\title{
NORMAL BUNDLES OF RATIONAL CURVES IN PROJECTIVE SPACES*
}

\author{
ZIV RAN ${ }^{\dagger}$
}

\begin{abstract}
We determine the splitting (isomorphism) type of the normal bundle of a generic genus-0 curve with 1 or 2 components in $\mathbb{P}^{n}$, as well as the way the bundle deforms locally with a general deformation of the curve. We deduce an enumerative formula for divisorial loci of smooth rational curves whose normal bundle is of non-generic splitting type.
\end{abstract}

Key words. Rational curve, projective space, normal bundle, enumerative geometry, cohomology theory, parameter spaces

AMS subject classifications. 14N10, 14N05

Rational curves in projective space, being essentially the same thing as finitedimensional vector spaces of rational functions in one variable, are among the most elementary and classical objects in Algebraic Geometry. In recent years it has become clear that suitable (compact) parameter spaces, say $R_{n, d}$, for rational curves of given degree $d$ in $\mathbb{P}^{n}$, are of fundamental importance. Now the geometry of a moduli or parameter space like $R_{n, d}$ is closely related to 'modular' subvarieties, i.e. ones defined in terms of the (universal) family of curves (or other objects) that it parametrizes. There are, to be sure, various ways of defining modular subvarieties of $R_{n, d}$, for instance the much-studied incidence subvarieties, parametrizing curves incident to a given cycle in $\mathbb{P}^{n}$. Another type of modular subvarieties involves vector bundles. Namely, given a 'reasonable function' $\Phi$ assigning to a curve $C \in R_{n, d}$ a vector bundle $E_{C}$ on $C$, a theorem of Grothendieck says we have a decomposition

$$
E_{C} \simeq \bigoplus \mathcal{O}_{C}\left(k_{i}\right), k_{1} \geq k_{2}, \ldots
$$

where $\mathcal{O}_{C}(k)$ denotes the unique line bundle of degree $k$ on $C$. The sequence $k$. $=$ $k .(C)$, which is uniquely determined and called the splitting type of $E_{C}$, varies uppersemicontinuously, in an obvious sense, in terms of the vector bundle and hence for a good function $\Phi$ we get a stratification $R_{n, d}^{\Phi}\left(k\right.$.) of $R_{n, d}$ where the strata consist of the curves $C$ with given sequence $k .(C)$.

One way to define an interesting, and reasonable, function $\Phi$ is to fix a vector bundle $E$ on $\mathbb{P}^{n}$ and to set

$$
E_{C}=\left.E\right|_{C} .
$$

The resulting stratification was studied in [R5] where we computed enumeratively its divisorial stratum. The main result of this paper is an analogous computation in the case where $\Phi$ is the 'normal bundle function', which assigns to a curve $C$ its normal bundle

$$
N_{C}=N_{C / \mathbb{P}^{n}}
$$

The splitting type $k .(C)$ of $N_{C}$, which we call the normal type of $C$, is a natural global numerical invariant of the embedding $C \subset \mathbb{P}^{n}$, perhaps the most fundamental such

* Received September 7, 2005; accepted for publication September 7, 2006.

$\dagger$ Department of Mathematics, University of California, Riverside, CA 92521, USA (ziv@math. ucr.edu). Partially supported by NSA Grant MDA904-02-1-0094. 
invariant beyond the degree, and thus the problem of enumerating curves with given normal type seems a natural one. Despite the existence of a natural surjection from the restricted tangent bundle

$$
\left.T_{\mathbb{P}^{n}}\right|_{C} \rightarrow N_{C},
$$

it turns out that in reality, there is little relationship between the splitting types of these bundles. Consequently, though the main enumerative result of this paper (Theorem 9.1) is an analogue for normal bundles of the result of [R5] for restricted bundles, there is in reality little of substance in common between the two papers.

In very broad outline, the proof of Theorem 9.1 goes as follows. To begin with, for the enumerative question to make sense it is necessary that the normal type $k .(C)$ of the generic curve in $R_{n, d}$ be 'well behaved'. This turns out to mean that this type is almost balanced in the sense that

$$
k_{1}(C)-k_{n-1}(C) \leq 1 .
$$

Assuming this, we need that the 'jump' of $k .(C)$ (from its generic value) occurs in codimension 1 . For that, it turns out that a necessary and sufficient condition is that $(d, n)$ be a perfect pair in the sense that

$$
(n-1) \mid 2 d-2,
$$

or equivalently, that the type $k .(C)$ of the generic $C$ should be balanced, in the sense that $k_{1}(C)=k_{n-1}(C)$. Fixing a perfect pair $(d, n)$, we may, as in [R5], consider a generic incidence pencil, i.e. an $\left(\infty^{1}\right) B$ of curves in $R_{n, d}$ defined by conditions of incidence to a generic collection of linear spaces, and the (smooth) surface $X$ swept out by the curves in $B$. On $X$ we may consider an appropriate vector bundle $G$ that is a twist of the 'relative lci normal bundle'. Then for smooth members of the pencil, unbalanced type can be interpreted in terms of the cohomology of $G$ : specifically as the local length of a suitable $R^{1}$ sheaf, which is of finite support and length, and where the corresponding $R^{0}$ vanishes. Now the Grothendieck-Riemann-Roch formula gives an expression for the total length of $R^{1}$. Therefore to complete the proof it 'only' remains to evaluate the contribution from singular, i.e. reducible fibres.

The evaluation of the local $R^{1}$ at the reducible fibres turns out, in reality, to be possibly the most involved part of the story. For, in contrast with the case of restricted bundles, the (lci) normal bundle of a reducible curve is quite often ill behaved; viz. there are natural notions of balanced and almost balanced for bundles on (2-component) reducible curves, and the normal bundle to a curve with a degenerate component is often not almost balanced (and even in cases when the latter bundle is almost balanced, that fact is relatively subtle to prove). This unbalancedness has some significant implications. First, it makes substantially more difficult the task of proving generic almost balancedness by specialization; second, and more consequentially, it means that in a pencil as above it is not sufficient to compute the cohomology on reducible fibres, but one must compute it in a neighborhood as well, i.e. compute the (length of) the entire local $R^{1}$ module, a substantially more painful computation. Painful or not, this computation does ultimately have a happy ending: its end result shows that $R^{1}$ is 'as small as possible', given the $H^{1}$ on the reducible fibre, i.e. that $R^{1}$ is killed by the maximal ideal. This result, that we call 'cohomological quasitransversality', is established by constructing an explicit smoothing of 'binomial' 
type for which the requisite property reduces to a combinatorial property of the exponents that we establish by a somewhat drawn-out, but quite elementary combinatorial argument. We hope this argument may find some other applications elsewhere.

The paper is organized as follows. In $\S 1$ we discuss elementary modifications of vector bundles and some of their basic properties. In $\S 2$ we discuss the (lci) relative normal bundle in a family of curves in a smooth ambient variety. In $\S 3$ we give a preliminary elementary discussion of normal bundles to rational curves, especially the rational normal curve, in projective space. In $\S 4$ we study in further detail the normal bundle to a rational normal curve and give a geometric interpretation of its splitting. In $\S 5$ we give a general elementary discussion of vector bundles on rational trees, especially 2-component trees that we call rational angles. On a rational angle, every vector bundle is a direct sum of lines bundles, but this fails on a general rational tree by Example 5.6. We study especially almost balanced bundles and their deformations and specializations. In $\S 6$ we give a complete determination of the normal bundles of general rational curves and rational angles in $\mathbb{P}^{n}$ (see Thm. 6.1). We find that the normal bundle is almost balanced for general rational curves of degree $d \geq n$; for a general rational angle $C_{a} \cup C_{b}$ we find that the normal bundle is almost balanced if both $a, b \geq n$ but usually not otherwise. After the paper was written, we found out that the case of smooth rational curves was known previously, due to Sacchiero [S], but the more general case of rational angles is apparently new, and is needed. $\S 7$ is preparatory to $\S 8$ in which we prove the cohomological quasitransversality result mentioned above. This $\S 8$ is perhaps the most technically involved part of the paper. After all these preparations, the proof of the main enumerative result, Theorem 9.1, is a straightforward adaptation of that of the main result of [R5], and like it uses the intersection calculus on incidence pencils, developed in earlier papers and reviewed in an Appendix.

An interesting question not settled by our work is that of irreducibility of the locus of curves of degree $d$ in $\mathbb{P}^{n}, n \geq 4$, with given normal type $(k$.). This seems open even in case $(d, n)$ is a perfect pair, so that the locus in question is of codimension 1 . For $n=3$ irreducibility is known by [EV2]. By contrast, the analogous irreducibility property for the restricted tangent bundle holds trivially, because, by the Euler sequence, the locus in question is parametrized by an open subset of the vector space

$$
\operatorname{Ext}^{1}\left(\bigoplus \mathcal{O}_{\mathbb{P}^{1}}\left(k_{i}\right), \mathcal{O}_{\mathbb{P}^{1}}\right)=H^{1}\left(\bigoplus \mathcal{O}_{\mathbb{P}^{1}}\left(-k_{i}\right)\right)
$$

(see also $[$ Ram $])$.

Acknowledgment. Some of this work was carried out while I was visiting the IHES, and I would like to thank the Institute and its staff for providing an ideal working environment. I also thank the organizers of the conference 'Current Geometry', held in Naples in June 2003, for an opportunity to present this work.

Notational conventions. On a nonsingular rational curve $C$ we will denote by $\mathcal{O}_{C}(k)$ or $\mathcal{O}(k)$ the unique line bundle of degree $k$ on $C$. On a reducible curve, we will use notation like $\mathcal{O}(j \cup k)$ or sometimes $\mathcal{O}(j, k)$ to denote the unique line bundle having degrees $j, k$ on the respective components. For any sheaf or abelian groups $L$ and natural number $m, m L$ usually denotes $\bigoplus_{1}^{m} L$ (unless it's clear that $L$ is being viewed as a divisor, in which case $m L$ is its multiple as such). We will be working 
over the groundfield $k=\mathbb{C}$, and using only closed points $p$, so that the residue field $k(p) \simeq \mathbb{C}$ always, and it is viewed as a skyscraper sheaf at $p$. For a coherent sheaf $L$ and a point $p$, the fibre $L(p):=L \otimes k(p)$.

1. Elementary Modifications. Let $X$ be a reduced algebraic scheme, $E$ a locally free coherent sheaf on $X$ and $\Sigma$ a Cartier divisor on $X$. Consider a quotient of the form

$$
E \stackrel{\phi}{\rightarrow} q \rightarrow 0
$$

where $q$ is a locally free $\mathcal{O}_{\Sigma}$-module. It is easy to see that the kernel $E^{\prime} \subset E$ of $\phi$ is a locally free sheaf; it is called the elementary reduction of $E$ corresponding to $q$, and (abusively) denoted $M(E, q)$ (of course in reality, $E^{\prime}$ depends on $\phi$ and not just $q$ ). We will primarily be interested in the cases

(1) $\mathrm{X}$ is a curve, $\Sigma$ is a reduced smooth point on $X$ and $q$ has length 1 ;

(2) $\mathrm{X}$ is a smooth surface and $\Sigma$ is a reduced curve on $X$.

From the defining exact sequence

$$
0 \rightarrow M(E, q) \rightarrow E \rightarrow q \rightarrow 0
$$

it is easy to compute the Chern classes of $M(E, q)$; e.g. in case (1) we get

$$
c_{1}(M(E, q))=c_{1}(E)-\ell(q) .
$$

Dualizing (1.2), we obtain the exact sequence

$$
0 \rightarrow E^{*} \rightarrow M(E, q)^{*} \rightarrow \sigma^{*} \rightarrow 0
$$

where $\sigma$ (and $S$ below) are defined by the exact diagram

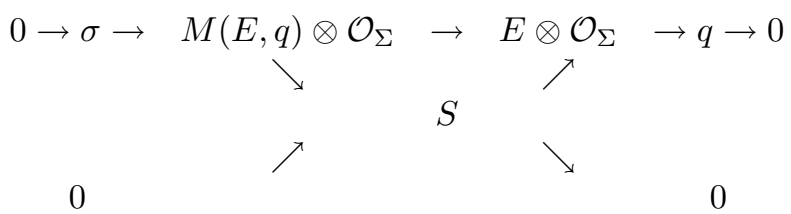

and $\sigma^{*}$ means $\mathcal{O}_{\Sigma}-$ dual. Thus

$$
M\left(M(E, q)^{*}, \sigma^{*}\right)^{*}=E .
$$

The bundle $M(E, q)^{*}$ may be called the elementary enlargement of $E^{*}$ corresponding to the subbundle $q^{*} \subseteq E^{*} \otimes \mathcal{O}_{\Sigma}$. An elementary modification is an elementary reduction or enlargement. It is also easy to see from (1.3) that

$$
M(M(E, q), S)=E(-\Sigma) .
$$

Consequently, an elementary reduction of $E$ is an elementary enlargement of $E(-\Sigma)$. In practice this means that it suffices to work with elementary reductions, which are more convenient than enlargements.

Now suppose we have an exact (locally split) sequence of vector bundles

$$
0 \rightarrow F \rightarrow E \rightarrow G \rightarrow 0 .
$$


We will say that $F$ survives (resp. gets chopped) in the elementary modification (1.1) if the induced map

$$
F \rightarrow q
$$

is zero (resp. surjective). If $F$ survives, then considering $F$ as subsheaf of $E$, we have

$$
F \otimes \mathcal{O}_{\Sigma} \subset S
$$

while, considering $F$ as subsheaf of $E^{\prime}=M(E, q)$, we have

$$
F \otimes \mathcal{O}_{\Sigma} \cap \sigma=0
$$

and we have an exact sequence

$$
0 \rightarrow F \rightarrow E^{\prime} \rightarrow G^{\prime} \rightarrow 0
$$

where $G^{\prime}=M(G, q)$. If $F$ gets chopped, we get a subbundle (i.e. locally split subsheaf)

$$
F^{\prime}:=M(F, q) \rightarrow E^{\prime}
$$

and

$$
\sigma \subseteq F^{\prime} \otimes \mathcal{O}_{\Sigma}
$$

and we have an exact sequence

$$
0 \rightarrow F^{\prime} \rightarrow E^{\prime} \rightarrow G \rightarrow 0 .
$$

2. The lci normal bundle. Let $\pi: X \rightarrow B$ be a family of nodal curves, $L$ a line bundle on $X$ and $f: X \rightarrow Y$ a generically 1-1 map to a smooth variety. Assume that $f$ is unramified on all fibres and an embedding on almost all fibres, including all singular ones. We have an exact sequence

$$
f^{*} \Omega_{Y}(L) \stackrel{d f}{\rightarrow} \omega_{X / B}(L) \rightarrow q_{\Sigma} \rightarrow 0
$$

where $\Sigma$ is the critical locus, i.e. the locus of singular points of fibres of $\pi$, and $q_{\Sigma}$ is a skyscraper sheaf with length 1 at each point of $\Sigma$. We denote $\operatorname{ker}(d f)$ by $N_{f / B}^{*}$ or just $N^{*}$ for short, so that we have a basic exact sequence

$$
0 \rightarrow N^{*}(L) \rightarrow f^{*} \Omega_{Y}(L) \stackrel{d f}{\rightarrow} \omega_{X / B}(L) \rightarrow q_{\Sigma} \rightarrow 0,
$$

where the image of $d f$ coincides with $\mathcal{I}_{\Sigma} \omega_{X / B}(L)$. In light of the exact sequence

$$
0 \rightarrow N^{*}(L) \rightarrow f^{*} \Omega_{Y}(L) \stackrel{d f}{\rightarrow} \Omega_{X / B}(L) \rightarrow 0,
$$

we see that $\Omega_{X / B}=\mathcal{I}_{\Sigma} \omega_{X / B}$, and in particular $\Omega_{X / B}$ is torsion-free and $\omega_{X / B}$ is its double dual. 
For any line bundle $L_{Y}$ on $Y$ and $L=f^{*} L_{Y}$, note the exact diagram

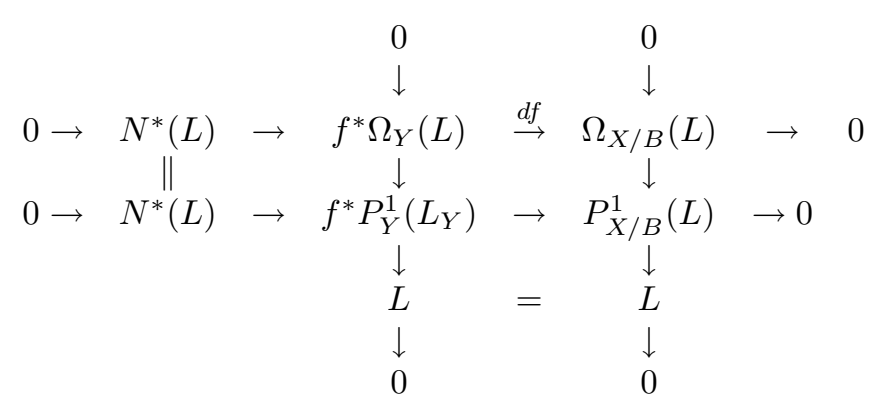

Here $P_{X / B}^{1}, P_{Y}^{1}$ denote the relative and absolute principal parts sheaves, reviewed in $\S 3$ below.

Let $P_{X / B}^{+}(L)$ be the double dual of $P_{X / B}^{1}(L)$, which may be called the sheaf of 'relative dualizing principal parts' of $L$, and which is clearly locally free and fits in a diagram

$$
\begin{aligned}
& 0 \rightarrow \Omega_{X / B}(L) \rightarrow P_{X / B}^{1}(L) \rightarrow L \quad \rightarrow 0 \\
& 0 \rightarrow \begin{array}{c}
\downarrow \\
\omega_{X / B}(L)
\end{array} \rightarrow P_{X / B}^{+}(L) \rightarrow \stackrel{\|}{L} \rightarrow 0 .
\end{aligned}
$$

Then we get an exact sequence

$$
0 \rightarrow N^{*}(L) \rightarrow f^{*}\left(P_{Y}^{1}\left(L_{Y}\right)\right) \rightarrow P_{X / B}^{+}(L) \rightarrow q_{\Sigma}(L) \rightarrow 0
$$

This sequence is especially useful when $Y=\mathbb{P}^{n}=\mathbb{P}(V)$ and $L_{Y}=\mathcal{O}(1)$-the case of principal interest to us- in which we have, as is well known $P_{Y}^{1}\left(L_{Y}\right)=V^{*} \otimes \mathcal{O}_{Y}$.

Now by easy and well known local computations (partly reproduced below), $N^{*}$ is a locally free sheaf of rank $n-1:=\operatorname{dim}(Y)-1$ and is called the (relative) lci conormal bundle of the map $f$. For any fibre $X_{b}$ we have, setting $C=f\left(X_{b}\right)$,

$$
N_{X_{b}}^{*} \simeq f^{*}\left(\mathcal{I}_{C} / \mathcal{I}_{C}^{2}\right) /(\text { torsion }) \text {. }
$$

In particular, if $\left.f\right|_{X_{b}}$ is an embedding, then

$$
N_{X_{b}}^{*} \simeq f^{*}\left(\mathcal{I}_{C} / \mathcal{I}_{C}^{2}\right)
$$

generally, if $x \in X_{b}$ is a smooth point then the fibre of $N^{*}$ at $x$, denoted $N^{*}(x)$, is canonically isomorphic to the conormal space at $f(x)$ of the unique branch of $C$ coming from an analytic neighborhood of $x$ on $X_{b}$.

Let us analyze the situation locally at a fibre node $p \in \Sigma$. For simplicity we assume the fibre $X_{0}$ through $p$ is a union of 2 smooth fibres $X_{1}, X_{2}$ - this is the case we will need. We may choose local coordinates $x_{1}, \ldots, x_{n}$ on $Y$ so that $X_{i}$ maps to the $x_{i}$-axis, $i=1,2$ so $C=f\left(X_{0}\right)$ is locally defined by

$$
x_{1} x_{2}=x_{3}=\cdots=x_{n}=0 .
$$

Let

$$
C_{i}=f\left(X_{i}\right), N_{i}^{*}=f^{*}\left(\mathcal{I}_{C_{i}} / \mathcal{I}_{C_{i}}^{2}\right), i=1,2 .
$$


Then we have an exact sequence

$$
\left.0 \rightarrow N^{*}\right|_{X_{1}} \rightarrow f^{*}\left(\mathcal{I}_{C_{1}} / I_{C_{1}}^{2}\right) \rightarrow q_{p} \rightarrow 0
$$

which restricts at $p$ to

$$
0 \rightarrow<\sigma_{p}>\rightarrow N^{*}(p) \rightarrow N_{1}^{*}(p) \rightarrow q_{p} \rightarrow 0 .
$$

In terms of bases, the latter sequence can be written

$$
\begin{gathered}
0 \rightarrow<x_{1} x_{2}>\rightarrow<x_{1} x_{2}, x_{3}, \cdots, x_{n}>\rightarrow \\
<x_{2}, \cdots, x_{n}>\rightarrow<x_{2}, \cdots, x_{n}>/<x_{3}, \cdots, x_{n}>\rightarrow 0 .
\end{gathered}
$$

We usually set $M=N^{*}(p)$ and $\sigma=\sigma_{p}$ is called the singular element of $M$ (welldefined up to scalar). Note that the image $S$ of $N^{*}(p) \rightarrow N_{1}^{*}(p)$, i.e. $\left\langle x_{3}, \cdots, x_{n}\right\rangle$, is just the Zariski conormal space to $C$ at $f(p)$, and we have an exact sequence of vector spaces

$$
0 \rightarrow<\sigma>\rightarrow M \rightarrow S \rightarrow 0
$$

Example 2.1. Let $C=L_{1} \cup L_{2}$ where $L_{1}, L_{2} \subset \mathbb{P}^{n}$ are distinct lines meeting at $p$. Then

$$
\left.N_{C}^{*}\right|_{L_{1}}=M\left(N_{L_{1}}^{*}, q\right)
$$

where $q$ is the quotient of $N_{L_{1}}^{*}$ corresponding to the tangent direction of $L_{2}$ at $p$. Since

$$
N_{L_{1}}^{*} \simeq N_{L_{1}}^{*}(p) \otimes \mathcal{O}_{L_{1}}(-1),
$$

it has a unique subsheaf $P_{1}$ that is isomorphic to $(n-2) \mathcal{O}(-1)$, and survives in $\left.N_{C}^{*}\right|_{L_{1}}=M\left(N_{L_{1}}^{*}, q\right)$, viz. $P_{1}=S \otimes \mathcal{O}(-1)$ (this notation means the obvious thing, that is, that $P_{1}$ isomorphic to $(n-2) \mathcal{O}(-1)$ and its fibre at $p$ is $\left.S \subset N_{1}^{*}(p)\right)$. Choosing any subsheaf of $N_{L_{1}}^{*}$ isomorphic to $\mathcal{O}(-1)$ and complementary to $P_{1}=S \otimes \mathcal{O}_{L_{1}}(-1)$, this subsheaf automatically gets chopped in $M\left(N_{L_{1}}^{*}, q\right)$ and yields a subbundle

$$
\left.Q_{1} \simeq \mathcal{O}_{L_{1}}(-2) \subset N_{C}^{*}\right|_{L_{1}}
$$

whose fibre at $p$ is $q$. Since the fibre at $p$ of $P_{1}$ is $S$ and $q \not \subset S$, we get a splitting

$$
\left.N_{C}^{*}\right|_{L_{1}} \simeq(n-2) \mathcal{O}_{L_{1}}(-1) \oplus \mathcal{O}_{L_{1}}(-2)=P_{1} \oplus Q_{1}
$$

in which $P_{1}$, and the fibre at $p$ of $Q_{1}$ (but not $Q_{1}$ itself), are uniquely determined, the latter being $q$. As already observed, the fibre at $p$ of the $P_{1}$ summand is just the Zariski conormal space which, in local coordinates as above, has $x_{3}, \ldots, x_{n}$ as basis.

Now $\left.N^{*}\right|_{L_{2}}$ can be analyzed similarly, yielding a decomposition

$$
\left.N_{C}^{*}\right|_{L_{2}} \simeq(n-2) \mathcal{O}_{L_{2}}(-1) \oplus \mathcal{O}_{L_{2}}(-2)=P_{2} \oplus Q_{2}
$$

and the fibres of $P_{2}, Q_{2}$ at $p$ are again $S, \sigma$, respectively. Therefore $P_{1}$ glues to $P_{2}$ and $Q_{1}$ to $Q_{2}$, yielding a splitting

$$
N_{C}^{*}=(n-2) \mathcal{O}((-1) \cup(-1)) \oplus \mathcal{O}((-2) \cup(-2))
$$

where $\mathcal{O}(a \cup b)$ is the line bundle on $C$ having degree $a$ on $L_{1}$ and $b$ on $L_{2}$ (such notation will be used throughout the paper). This result if of course obvious from the 
fact that $C$ is a $\left(2,1^{n-2}\right)$ complete intersection, but is nonetheless enlightening in that it shows that the two positive subsheaves

$$
\left.P_{i} \subset N_{C}^{*}\right|_{L_{i}}, i=1,2
$$

are not mutually in general position at $p$, contrary to what one might naively have expected. This contrasts with the situation when normal bundles are replaced by restrictions of a fixed (and suitable) bundle on $\mathbb{P}^{n}$, for instance the tangent bundle (cf $[R 5], \S 2)$.

To formalize the sort of situation typically encountered in analyzing the normal bundle of a reducible curve, it is convenient to introduce some definitions.

DeFINITION 2.2. Let $M$ be a vector space with a distinguished 1-dimensional subspace $\langle\sigma\rangle$. A pair F., G. of increasing filtrations on $M$ are said to be in relative general position (with respect to $\sigma$, if that is not understood) provided we have for each $i, j$ that

(i) whenever $\sigma \notin F_{i} \cap G_{j}, F_{i}$ and $G_{j}$ are in general position in $M$;

(ii) whenever $\sigma \in F_{i} \cap G_{j}, F_{i} / \sigma, G_{j} / \sigma$ are in general position in $M / \sigma$.

Now let $X_{1}, X_{2} \subset \mathbb{P}^{n}$ be a pair of smooth curves meeting transversely at a point $p$, and set

$$
X=X_{1} \cup X_{2}, M=N_{X}^{*} \otimes k(p)
$$

with $\sigma \in M$ the singular element. For any vector bundle $E$ on a smooth curve $C$, we denote by $H N .(E)$ the (increasing) Harder-Narasimhan filtration of $E$ and, for any point $p \in C$, by $H N .(E, p)$ the fibre of the latter at $p$, i.e. $H N .(E) \otimes k(p)$. We refer to $H N_{1}(E)$ as the positive subsheaf of $E$ and denote it by $E_{+}$, and to $H N_{1}(E, p)$ as the positive subspace of $E(p)=E \otimes k(p)$.

Definition 2.3. $X_{1}$ and $X_{2}$ are said to have good interface (or to interface well) at $p$ if the filtrations

$$
H N_{\bullet}\left(\left.N_{X}^{*}\right|_{X_{i}}, p\right) \subseteq M, i=1,2
$$

are in relative general position.

For instance, in the above example we showed a transverse pair of lines $L_{1}, L_{2}$ in $\mathbb{P}^{n}$ do not interface well (at their point of intersection). This is closely related to the fact that $L_{1} \cup L_{2}$ is not almost balanced. Indeed the following general remark is easy to prove

Lemma 2.4. Let $X_{1}, X_{2}$ be a general pair of rational curves in $\mathbb{P}^{n}$ meeting at $p$. Assume both are almost balanced. Let $X=X_{1} \cup X_{2}$. Then

(i) $\left.N_{X}^{*}\right|_{X_{i}}$ is almost balanced for $i=1,2$

(ii) $X_{1}, X_{2}$ have good interface at $p$ iff the fibres at $p$ of the positive subbundles of $\left.N_{X}^{*}\right|_{X_{i}}, i=1,2$ meet transversely in $N_{X}^{*}(p)$.

Indeed (i) follows from the easy fact that for an almost balanced bundle $E$ on $\mathbb{P}^{1}$ and a point $p \in \mathbb{P}^{1}$, the elementary reduction of $E$ corresponding to a general quotient of $E(p)$ (see $\S 1$ ) is almost balanced. Assertion (ii) follows from Proposition 5.3(ii) below.

In the case where the ambient space is $\mathbb{P}^{n}$ it will often be convenient to work with the twisted bundles $N \otimes L^{-1}, N^{*} \otimes L$, where $L=f^{*} \mathcal{O}_{\mathbb{P} n}$ (1) (which might be called 
the normalized normal and conormal bundles), and it will be convenient to use the notation

$$
\mathbb{N}=N \otimes L^{-1}, \mathbb{N}^{*}=N^{*} \otimes L .
$$

3. The osculatrix filtration. For a smooth scheme $X / B$, and a (say locally free) sheaf $L$ on $X$, we denote by $P_{X / B}^{m}(L)$ or just $P^{m}(L)$ the sheaf of $m$ th order principal parts of $L$ (cf. [ EGA]). This sheaf carries a natural increasing filtration with the $i$ th quotient being $P^{i}(L)$ and the $i$ th graded piece being $\operatorname{Sym}^{i}\left(\Omega_{X / B}\right) \otimes L$. We will denote the $i$ th subsheaf in this filtration, i.e. the kernel of the natural map

$$
P^{m}(L) \rightarrow P^{i}(L)
$$

by $P^{[m, i)}(L)$.

Example 3.1. If $X=\mathbb{P}^{n}, L=\mathcal{O}(1), V=H^{0}(L)$, the canonical map

$$
V \otimes \mathcal{O}_{X} \rightarrow P^{1}(L)
$$

is easily seen to be an isomorphism.

Now let $C \rightarrow \mathbb{P}^{n}$ be a smooth curve and again let $V=H^{0}\left(\mathcal{O}_{\mathbb{P}^{n}}(1)\right), L=\mathcal{O}_{C}(1)$. We have a natural map

$$
\rho: V \otimes \mathcal{O}_{C} \rightarrow P_{C}^{n-1}(L) .
$$

Note that the 'expected degeneracy' of $\rho$ is in codimension 2. We will say that $C$ is totally unramified if $\rho$ is surjective.

Now suppose that $C$ is totally unramified and set, as usual

$$
M=M_{C}=\Omega_{\mathbb{P}^{n}}^{1}(1) \otimes \mathcal{O}_{C} .
$$

Then in light of Example 3.1 $M$ coincides with the kernel of the natural evaluation map

$$
V \otimes \mathcal{O}_{C} \rightarrow L
$$

hence we get a natural map

$$
\rho: M \rightarrow P^{[n, 0)}(L), P=P_{C},
$$

inducing a surjection

$$
M \rightarrow P^{[n-1,0)}(L)
$$

Set

$$
M^{i}=\rho^{-1}\left(P^{[n, i)}(L)\right), i=0, \ldots, n-1 .
$$

Then $(M \cdot)$ is a descending filtration of $M$ with

$$
M^{i-1} / M^{i}=K^{i} \otimes L, 1 \leq i \leq n-1,
$$

where $K=\omega_{C}$, while $M^{n-1}$ is a subsheaf, usually proper, of $K^{n} \otimes L$. Note that by (3.1),

$$
M^{n-1} \otimes L^{n-1} \otimes K^{\left(\begin{array}{c}
n \\
2
\end{array}\right)}=\operatorname{det}(M)=L^{-1}
$$

and hence

$$
M^{n-1}=L^{-n} \otimes K^{-\left(\begin{array}{c}
n \\
2
\end{array}\right)} .
$$


Note that by definition $M^{1}$ coincides with the twisted conormal sheaf $N^{*}(L)$, whose fibre at any point $p \in C$ is the set of linear forms vanishing on the embedded tangent line

$$
T_{p} C \subset \mathbb{P}^{n} .
$$

Similarly the fibre of $M^{i}$ at $p$ for $i \leq n-1$ coincides with the set of linear forms vanishing on the $i$ th tangent (or $(i-1)$ st osculating) space $T_{p}^{i} C$, i.e. the set of linear forms vanishing to order at least $i+1$ at $p$ (the latter may be taken as the definition of $T_{p}^{i} C$ ); by our assumption of total non-ramification $T_{p}^{i} C$ has constant dimension for all $p \in C, i \leq n-1$ and if $i<n-1$ then $T_{p}^{i} C$ osculates to $C$ to order exactly $i+1$.

Example 3.2: The Rational Normal Curve. Let

$$
C=C_{n} \subset \mathbb{P}^{n}
$$

be the rational normal curve. Thus

$$
V=H^{0}\left(\mathcal{O}_{C}(n)\right)=: V_{n},
$$

hence the map

$$
V \otimes \mathcal{O}_{C} \rightarrow P^{n}\left(\mathcal{O}_{C}(n)\right)
$$

is fibrewise injective ('a degree- $n$ polynomial is determined by its $n$-th order Taylor expansion at any point') hence this map is in fact an isomorphism. Likewise, we may identify the fibre of $M^{i}$ at $p \in C$ with

$$
H^{0}\left(\mathcal{O}_{C}(n-(i+1) p)\right)=H^{0}\left(\mathcal{O}_{C}(n-i-1)\right),
$$

an identification which, up to scalars, is independent of the point $p \in C$. It follows that

$$
M^{i} \simeq V_{n-1-i} \otimes L_{i}
$$

where $V_{n-1-i}=H^{0}\left(\mathcal{O}_{C}(n-i-1)\right)$ is an $(n-i)$-dimensional vector space and $L_{i}$ is some line bundle on $C$. Comparing degrees via (3.1) we see that $L_{i}$ has degree $-i-1$, i.e.

$$
M^{i} \simeq V_{n-1-i} \otimes \mathcal{O}_{C}(-i-1) .
$$

Alternatively, and more directly, one may observe that given $p \in C \simeq \mathbb{P}^{1}$ and a linear form $\ell_{p}$ on $\mathbb{P}^{1}$ zero at $p$, elements of the fibre at $p$ of $V_{n-1-i} \otimes \mathcal{O}_{C}(-i-1)$ may be represented uniquely in the form $f . \ell_{p}^{i+1}, f \in V_{n-1-i}$ which gives rise to a natural inclusion

$$
V_{n-1-i} \otimes \mathcal{O}_{C}(-i-1) \rightarrow V_{n}
$$

the image of this inclusion at $p$ clearly coincides with the set of polynomials whose $i$ th jet at $p$ is zero, thus the image globally coincides with $M^{i}$, so $M^{i} \simeq V_{n-1-i} \otimes \mathcal{O}_{C}(-i-1)$ and we have exact sequences

$$
0 \rightarrow V_{n-1-i} \otimes \mathcal{O}_{C}(-i-1) \rightarrow V_{n} \otimes \mathcal{O}_{C} \rightarrow P^{i}\left(\mathcal{O}_{C}(n)\right) \rightarrow 0 .
$$

In particular for $i=1$ we deduce the isomorphism

$$
N_{C_{n}}^{*} \simeq V_{n-2} \otimes \mathcal{O}_{C}(-n-2)
$$


Note that under the above identification the inclusion $M^{i} \subset M^{j}$ is given at $p \in C$ by

$$
f \mapsto f \ell_{p}^{j-i}
$$

where $\ell_{p}$ is the unique, up to scalars, linear polynomial vanishing at $p$. From this it follows easily that for any $i>j$, the inclusion $M^{i} \subset M^{j}$ is 'nondegenerate' in the sense that its image is not contained in a 'flat' subbundle of the form

$$
W \otimes \mathcal{O}_{C}(-j-1), W \subsetneq V^{j} .
$$

Corollary 3.3. A general rational curve of degree $d$ in $\mathbb{P}^{n}$ is totally unramified.

Proof. We may assume the curve $C$ is nondegenerate, i.e. $d \geq n$. Then $C$ is obtained as the projection of a rational normal curve in $\mathbb{P}^{d}$ corresponding to a general $(n+1)$-dimensional subspace

$$
V \subset H^{0}\left(\mathcal{O}_{C}(d)\right)=: V_{d} .
$$

Recall the isomorphism

$$
V_{d} \otimes \mathcal{O}_{C} \simeq P^{d}\left(\mathcal{O}_{C}(d)\right)
$$

and the natural surjection

$$
P^{d}\left(\mathcal{O}_{C}(d)\right) \rightarrow P^{n-1}\left(\mathcal{O}_{C}(d)\right)
$$

Then $C$ being totally unramified is equivalent to $V$ mapping surjectively to $P^{n-1}$ $\left(\mathcal{O}_{C}(d)\right)$. That this holds for a general $V$ can be seen by a standard (and trivial) dimension count.

Example 3.4. Let $C_{d} \subset \mathbb{P}^{n}$ be a generic rational curve of degree $d \leq n$. Then $C_{d}$ is a rational normal curve within the span $\mathbb{P}^{d}$ of $C_{d}$. As is well known,

$$
\left.T_{\mathbb{P}^{n}}\right|_{\mathbb{P}^{d}} \simeq T_{\mathbb{P}^{d}} \oplus(n-d) \mathcal{O}(1)
$$

therefore

$$
\left.N_{C_{d}}^{*} \simeq N_{C_{d} / \mathbb{P}^{d}}^{*} \oplus(n-d) \mathcal{O}_{\mathbb{P}^{d}}(-1)\right|_{C_{d}}
$$

thus

$$
N_{C_{d}}^{*} \simeq(n-d) \mathcal{O}(-d) \oplus(d-1) \mathcal{O}(-d-2) .
$$

A fact closely related to the exact sequence (3.3) for $i=1$ is the following observation, which is probably well known, but of which we shall subsequently require a more precise from

Lemma 3.5. For $n>0$, there is a canonical isomorphism on $\mathbb{P}^{1}$

$$
\alpha: P^{1}(\mathcal{O}(n)) \simeq V_{1} \otimes \mathcal{O}(n-1)
$$

such that, denoting by

$$
\pi: V_{n} \rightarrow H^{0}\left(P^{1}(\mathcal{O}(n))\right)
$$

the usual isomorphism, the composite

$$
H^{0}(\alpha) \circ \pi: V_{n} \rightarrow V_{1} \otimes V_{n-1}
$$


coincides with the usual co-symmetrization map

$$
\ell_{1} \cdots \ell_{n} \mapsto \sum_{i} \ell_{i} \otimes \ell_{1} \cdots \hat{\ell}_{i} \cdots \ell_{n}
$$

Proof. In terms of homogeneous coordinates $X_{0}, X_{1}$, consider the map $\phi$ given by

$$
\phi(f)=X_{0} \otimes \partial f / \partial X_{0}+X_{1} \otimes \partial f / \partial X_{1} .
$$

Being a 1st-order differential operator in $f$, this expression clearly descends to a canonical $\mathcal{O}_{\mathbb{P}^{1}}$-linear, PGL-equivariant map

$$
P^{1}(\mathcal{O}(n)) \rightarrow V_{1} \otimes \mathcal{O}(n-1) .
$$

To show this map is an isomorphism it suffices to check it is surjective at one point, e.g. $[1,0]$, where this is obvious.

Remark 3.6. The fact that $P^{1}(\mathcal{O}(n)) \simeq 2 \mathcal{O}(n-1)$ also follows from the fact that the extension class of the natural exact sequence

$$
0 \rightarrow \Omega_{\mathbb{P}^{1}}(n) \rightarrow P^{1}(\mathcal{O}(n)) \rightarrow \mathcal{O}(n) \rightarrow 0
$$

represents the first Chern class of $\mathcal{O}(n)$, hence the sequence is nonsplit if $n \neq 0$. However, the explicit isomorphism given above will be important in the sequel (see especially Sect. 7).

4. Realizing the splitting geometrically. Continuing with the case of the rational normal curve $C=C_{n} \subset \mathbb{P}^{n}$, consider the special case of (3.2) that is the isomorphism

$$
N^{*}(L) \simeq V_{n-2} \otimes \mathcal{O}(-2)
$$

where $L=\mathcal{O}_{C}(n)=\left.\mathcal{O}_{\mathbb{P}^{n}}(1)\right|_{C}$ and

$$
N^{*}=M_{C}^{1}=\mathcal{I}_{C} / \mathcal{I}_{C}^{2}
$$

is the conormal bundle. This splitting of the conormal bundle may be realized geometrically as follows. Note that the set of divisors of degree $n-2$ on $C$ may be identified with $\mathbb{P}\left(V_{n-2}\right)$. Let $\mathfrak{D}$ be such a divisor. Projection from $\mathfrak{D}$, that is, from its (scheme-theoretic) linear span $\overline{\mathfrak{D}} \simeq \mathbb{P}^{n-3} \subset \mathbb{P}^{n}$, maps $C$ to a smooth conic in $\mathbb{P}^{2}$, whose pullback $K_{\mathfrak{D}}$, i.e. the cone on $C$ with vertex $\overline{\mathfrak{D}}$, is a rank-3 quadric in $\mathbb{P}^{n}$ containing $C$. Note that the map

$$
\mathfrak{D} \mapsto K_{\mathfrak{D}}
$$

is one-to-one because $\overline{\mathfrak{D}}$ coincides with the singular locus of $K_{\mathfrak{D}}$ while

$$
\mathfrak{D}=\overline{\mathfrak{D}} \cap C .
$$

The equation of $K_{\mathfrak{D}}$ gives rise to a nonzero section of $N^{*}(2 L)=N^{*}(2 n)$ vanishing on $\mathfrak{D}$. Untwisting by $\mathfrak{D}$, we get a subsheaf

$$
\kappa_{\mathfrak{D}} \simeq \mathcal{O}_{C}(-2) \subset N^{*}(L) .
$$

As $N^{*}(n+2)$ is a trivial bundle, $\kappa_{\mathfrak{D}}$ must be a (saturated) subbundle. Now the natural map

$$
H^{0}\left(\mathcal{I}_{C}(2 L)\right) \rightarrow H^{0}\left(N_{C}^{*}(2 L)\right)
$$


is clearly injective: indeed a nonzero element of its kernel would yield a quadric $K$ double along $C$,i.e. such that $C$ is contained in the singular locus of $K$; but the singular locus of any quadric must be a proper linear subspace of $\mathbb{P}^{n}$, hence cannot contain $C$, hence $K$ cannot exist. Thus the assignment

$$
\mathfrak{D} \mapsto \kappa_{\mathfrak{D}}
$$

is one-to one, giving rise to a one-to-one map

$$
\bar{\lambda}: \mathbb{P}\left(H^{0}\left(\mathcal{O}_{C}(n-2)\right)\right) \rightarrow \mathbb{P}\left(N_{C}^{*}(n+2)\right) .
$$

Since both source and target of $\lambda$ are $\mathbb{P}^{n-2}$ s, $\lambda$ must be a projective isomorphism, arising from a linear isomorphism

$$
\lambda: H^{0}\left(\mathcal{O}_{C}(n-2)\right) \rightarrow H^{0}\left(N_{C}^{*}(n+2)\right) .
$$

It would be nice to construct $\lambda$ directly as a linear map of vector spaces, but we don't know how to do it. In any event, it is clear by construction that for a general $p \in C$, the fibre $\kappa_{\mathfrak{D}}(p)$ corresponds to the hyperplane in $N_{C}(p)$ that comes from the hyperplane

$$
<T_{p} C, \mathfrak{D}>\subset \mathbb{P}^{n}
$$

and it follows easily from this that for a general choice of divisors $\mathfrak{D}_{1}, \ldots, \mathfrak{D}_{n-1}$ and a general $p \in C$, the subsheaves $\kappa_{\mathfrak{D}_{1}}, \ldots, \kappa_{\mathfrak{D}_{n-1}}$ are independent at $p$, whence a generically injective map

$$
\phi:(n-1) \mathcal{O}(-2) \rightarrow N^{*}(L)
$$

which, in view of (4.1), must be an isomorphism, giving the desired realization of the splitting (4.1). Note that it follows a posteriori that $\kappa_{\mathfrak{D}} \subset N^{*}(L)$ is a subbundle isomorphic to $\mathcal{O}_{C}(-2)$ for all divisors $\mathfrak{D}$, not just general ones. Note also that for any $\mathfrak{D}$ we may choose homogeneous coordinates $X_{0}, \ldots, X_{n}$ so that $\kappa_{\mathfrak{D}}$ is given off $\mathfrak{D}$ by

$$
F=X_{0} X_{2}-X_{1}^{2}
$$

or in terms of affine coordinates $x_{1}, \ldots, x_{n}$, by

$$
f=x_{2}-x_{1}^{2} \text {. }
$$

5. Vector bundles on some rational trees. In view of Grothendieck's theorem about decomposability of vector bundles on nonsingular rational curves, it is natural to ask to what extent decomposability holds for vector bundles on rational trees, i.e. nodal curves of arithmetic genus 0 . The following result is hardly surprising; it will not be used as such in the sequel, but the method of proof will.

Proposition 5.1. Let $C$ be a nodal curve of the form

$$
C=C_{1} \cup_{p} C_{2}
$$

where $C_{1}, C_{2}$ are nonsingular, rational, and meet only at $p$ (we call such a curve $C$ a rational angle). Then any locally free coherent sheaf on $C$ is a direct sum of line bundles.

Proof. We begin with the following observation. Let $F$ be a vector bundle on a nonsingular rational curve $D, q$ a point on $D$ and $v \in F(q)$ a nonzero element of the fibre at $q$. Then there is a basis of $F(q)$ that contains $v$ and is compatible with the 
filtration induced on $F(q)$ by the Harder-Narasimhan filtration $H N .(F)$. This is a triviality. It follows from it that there is a line subbundle $L \subset F$ so that $v \in L(q)$ and that

$$
F \simeq L \oplus(F / L)
$$

Indeed if $i$ is such that

$$
v \in H N_{i}(F)(q) \backslash H N_{i-1}(F),
$$

there is a unique line bundle summand $\bar{L}$ of $H N_{i}(F) / H N_{i-1}(F)$ such that

$$
v \in \bar{L}(q) \bmod H N_{i-1}(F),
$$

and we can take for $L$ any lifting of $\bar{L}$ to

$$
H N_{i}(F) \simeq H N_{i-1}(F) \oplus H N_{i}(F) / H N_{i-1}(F) \subseteq F .
$$

Now let $E$ be a vector bundle on $C$ as in the Proposition, and let $\left.E_{1} \subseteq E\right|_{C_{1}}$ be the positive subbundle, i.e. the smallest nonzero subsheaf in the Harder-Narasimhan filtration of $\left.E\right|_{C_{1}}$. Pick any

$$
0 \neq v \in E_{1}(p)
$$

and apply the above observation with $D=C_{2}, q=p, F=\left.E\right|_{C_{2}}$. It yields a line bundle summand $L \subset F$ with fibre at $p$ generated by $v$ and a complementary summand $G \subset F$. Set

$$
W=G(p) \subset E(p) .
$$

Now there is a line bundle summand $M$ of $\left.E_{1}\right|_{C_{1}}$ with fibre at $p$ generated by $v$, and clearly $M$ is also a summand of $\left.E\right|_{C_{1}}$. Thanks to the fact that $v \notin W$, it follows that $W$ is in general position with respect to the filtration on $E(p)$ induced by the HarderNarasimhan filtration of $\left.E\right|_{C_{1}}$. Hence there is a complementary subbundle $B$ to $M$ in $\left.E\right|_{C_{1}}$ with $B(p)=W$. Then $L, M$ glue to a line subbundle $\Lambda$ of $E$ and $G, B$ glue to a complementary subbundle $\Gamma$, with

$$
E \simeq \Lambda \oplus \Gamma .
$$

By induction on the rank of $E, \Gamma$ is a direct sum of lines bundles, hence so is $E$.

Remark 5.1.1. As we shall see, the method of proof of Proposition 5.1 can and will be used to construct explicit splittings on rational angles. However neither the statement of Proposition 5.1, nor for that matter that of Grothendieck's theorem, will be used.

Next, recall that a vector bundle $F$ on a nonsingular rational curve $D$ is said to be almost balanced if it has the form

$$
F=r_{+} \mathcal{O}(k) \oplus s \mathcal{O}(k-1)
$$

and balanced if we may moreover assume $s=0$. The subsheaf

$$
F_{+}=r_{+} \mathcal{O}(k) \subseteq F
$$

is then uniquely determined and called the positive subsheaf of $F$. The following remark, whose (trivial) proof we omit, gives a useful cohomological characterization of almost balanced bundles. 
Lemma 5.2. $F$ is almost balanced iff some twist $G$ of $F$ satisfies

$$
H^{1}(G)=0, \quad H^{0}(G(-1))=0 .
$$

A vector bundle $E$ on a rational angle $C=C_{1} \cup_{p} C_{2}$ is said to be almost balanced or $\mathrm{AB}$ if it has either the form

$$
E=r_{+} \mathcal{O}(k \cup \ell) \oplus s_{1} \mathcal{O}(k \cup \ell-1) \oplus s_{2} \mathcal{O}(k-1 \cup \ell)
$$

or the form

$$
E=r_{1+} \mathcal{O}(k+1 \cup \ell) \oplus r_{2+} \mathcal{O}(k \cup \ell+1) \oplus s \mathcal{O}(k \cup \ell)
$$

where $\mathcal{O}(a \cup b)$ denotes the lines bundle having degree $a$ on $C_{1}, b$ on $C_{2}$ (any line bundle on $C$ is one of these for a unique $(a, b)) ; E$ is balanced if we may further assume $r_{+}=0$ in (5.1) or $s=0$ in (5.2). A convenient characterization of $\mathrm{AB}$ bundles is the following

Proposition 5.3. Given a vector bundle $E$ on a rational angle, the following are equivalent:

(i) $E$ is almost balanced;

(ii)the bundles $E_{i}=E \mid C_{i}, i=1,2$ are almost balanced and the positive subspaces

$$
\left(E_{i}\right)_{+}(p) \subseteq E(p), i=1,2
$$

are in general position;

(iii) some twist $G=E(-k,-\ell)$ satisfies either

$$
H^{1}(G)=H^{0}(G(-1 \cup 0))=H^{0}(G(0 \cup-1))=0
$$

or

$$
H^{0}(G)=H^{1}(G(1 \cup 0))=H^{1}(G(0 \cup 1))=0
$$

Proof. The fact that (i) implies (ii) and (iii) is trivial. The proof that (ii) implies (i) is very similar to that of Proposition 5.1 (whether (5.3) or (5.3') occurs depends on whether the two positive subspaces $\left(E_{1}\right)_{+}(p),\left(E_{2}\right)_{+}(p)$ span $E(p)$ or not). To prove that (iii)- say in the form (5.3)- implies (i) we use Proposition 5.1. We may assume $E$ contains $\mathcal{O}(0 \cup 0)$ as a direct summand, in which case the $H^{0}$ vanishing hypotheses in (5.3) show that $E$ cannot have a direct summand $\mathcal{O}(a \cup b)$ with either $a>0$ or $b>0$, while the $H^{1}$ vanishing hypothesis implies that $E$ cannot have a direct summand $\mathcal{O}(a \cup b)$ with either $a<-1$ or $b<-1$ or $(a, b)=(-1,-1)$. Hence $E$ is a sum of copies of $\mathcal{O}(0 \cup 0), \mathcal{O}(0 \cup-1)$ and $\mathcal{O}(-1 \cup 0)$ so it is almost balanced.

Corollary 5.4. Suppose $\left(C_{t}, E_{t}\right)$ is a flat family of pairs (curve, vector bundle) such that $C_{0}$ is a rational angle, $E_{0}$ is almost balanced and a general $C_{t}$ is smooth. Then a general $E_{t}$ is almost balanced.

Remark 5.4.1. A family of pairs (curve, vector bundle) coming from a flat family $\mathcal{C} / T$ and a vector bundle $\mathcal{E}$ on $\mathcal{C}$ is said to be quasi-constant if there is a filtration of $\mathcal{E}$ with vector bundle quotients which restricts to the HN filtration on each fibre. Then the same argument shows that if $E_{0}$ is almost balanced then any family is quasi-constant: in fact, locally over the base, $\mathcal{E}$ itself splits as a direct sum of line bundles. 
Example 5.5: Piecewise degenerate rational angles. The argument in the proof of Proposition 5.1 may be used to construct splittings of vector bundles on rational angles. As an example, which will be needed in the sequel, we consider a general rational angle of the form

$$
C=C_{a, b}=C_{a} \cup C_{b} \subset \mathbb{P}^{n}
$$

where $C_{a}, C_{b}$ have degrees $a, b$ respectively with $a, b<n$ and they meet at $p$. Thus $C_{a}$ is a rational normal curve in $\mathbb{P}^{a}=<C_{a}>$ and likewise for $C_{b}$. Set

$$
N^{*}=N_{C}^{*} \text {. }
$$

Suppose first that $a+b \geq n$. Now clearly we have

$$
N_{C_{a}}^{*} \simeq(n-a) \mathcal{O}(-a) \oplus(a-1) \mathcal{O}(-a-2)
$$

and $\left.N^{*}\right|_{C_{a}}$ is obtained from this by an elementary modification at $p$ corresponding to $T_{p} C_{b}$, which is a general direction. This modification has the effect of chopping an $\mathcal{O}(-a)$ summand down to an $\mathcal{O}(-a-1)$, and it follows easily that

$$
\left.N^{*}\right|_{C_{a}} \simeq(n-a-1) \mathcal{O}(-a) \oplus \mathcal{O}(-a-1) \oplus(a-1) \mathcal{O}(-a-2) .
$$

The HN filtration of this sheaf is

$$
\begin{gathered}
H N_{1}\left(\left.N^{*}\right|_{C_{a}}\right)=(n-a-1) \mathcal{O}(-a), \\
H N_{2}\left(\left.N^{*}\right|_{C_{a}}\right)=(n-a-2) \mathcal{O}(-a) \oplus \mathcal{O}(-a-1) .
\end{gathered}
$$

Ditto for $C_{b}$. Let $M=N^{*}(p)$ and let

$$
\Phi_{i}^{c} \subset M, i=1,2, c=a, b
$$

be the fibres of these HN sheaves. Then $\Phi_{1}^{a}$ may be identified with the set of linear forms vanishing on $\mathbb{P}^{a} \cup T_{p} C_{b}$. In particular it does not contain the singular element $\sigma$ and its image in the Zariski conormal space

$$
S \simeq M /<\sigma>
$$

is a generic $(n-a-1)$-dimensional subspace and ditto for $b$. Since

$$
(n-a-1)+(n-b-1)<n-1=\operatorname{dim}(S)
$$

by our assumption $a+b \geq n$, these subspaces have zero intersection and in particular $\Phi_{1}^{a} \cap \Phi_{1}^{b}=0$. On the other hand, clearly

$$
\Phi_{2}^{a}=\Phi_{1}^{a} \oplus<\sigma>
$$

and likewise for $b$. Given this information, it is easy to see, arguing as in the proof of Proposition 5.1, that we have the following splitting (valid for $a+b \geq n$ )

$$
\begin{gathered}
N^{*} \simeq(n-a-1) \mathcal{O}((-a) \cup(-b-2) \oplus(n-b-1) \mathcal{O}((-a-2) \cup(-b)) \\
\oplus \mathcal{O}((-a-1) \cup(-b-1)) \oplus(a+b-n) \mathcal{O}((-a-2) \cup(-b-2)) .
\end{gathered}
$$

Note that if $a+b>n$ this bundle is never almost balanced.

Suppose next that

$$
a+b<n
$$


Then $\mathbb{P}^{a} \cup \mathbb{P}^{b}$ spans a $\mathbb{P}^{a+b}$ and we have a splitting

$$
N^{*} \simeq N_{C / \mathbb{P}^{a+b}}^{*} \oplus(n-a-b) L^{*}, L^{*}=\left.\mathcal{O}_{\mathbb{P}^{n}}(1)\right|_{C}=\mathcal{O}((-a) \cup(-b)) .
$$

Applying (5.5) to the inclusion $C_{a} \cup C_{b} \subset \mathbb{P}^{a+b}$, we get the following splitting case $a+b<n$ :

$$
\begin{aligned}
& N^{*} \simeq(n-a-b) \mathcal{O}((-a) \cup(-b)) \oplus(b-1) \mathcal{O}((-a) \cup(-b-2) \\
& \oplus(a-1) \mathcal{O}((-a-2) \cup(-b)) \oplus \mathcal{O}((-a-1) \cup(-b-1)) .
\end{aligned}
$$

Despite Proposition 5.1, it is not in general true that a vector bundle, even of rank 2 , on a rational tree is decomposable, as the following example shows.

Example 5.6. Consider a nodal curve of the form

$$
C=C_{0} \cup C_{1} \cup C_{2} \cup C_{3} \cup C_{4}
$$

where each component $C_{i}$ is a $\mathbb{P}^{1}, C_{0}$ meets each $C_{i}, i>0$, in a unique point $p_{i}$ and there are no other intersections. A vector bundle $E$ on $C$ may be constructed by taking a copy of $\mathcal{O}(1) \oplus \mathcal{O}$ on each component $C_{i}$ and gluing together generically at the $p_{i}$. Note that if a line subbundle $L$ of $E$ has degree 1 on some $C_{i}, i>0$ then it has degree 0 on $C_{0}$ and there is at most one other component $C_{j}$ on which $L$ has degree 1. Similarly, if $L$ has degree 1 on $C_{0}$ then it has degree 0 on every other $C_{i}$. It follows that any line subbundle of $E$ must have degree at most 2 and since $E$ has degree 5 it is indecomposable.

In general, when an almost balanced bundle specializes to a non-almost balanced one, there is no well-defined limit to the maximal subbundle. In the next result, however, we identify one very special case when the limit can be at least partly identified.

Proposition 5.7. Let $X / B$ be a proper family with general fibre $\mathbb{P}^{1}$ and special fibre $X_{0}$ either a $\mathbb{P}^{1}$ or a rational angle, and with $X$ a smooth surface and $B$ a smooth curve. Let $E$ be a vector bundle on $X$ whose restriction $E_{b}$ on a general fibre $X_{b}$ is almost balanced and whose restriction $E_{0}$ on $X_{0}$ admits a filtration

$$
E_{00}=0 \subseteq E_{01} \subseteq E_{02} \subseteq E_{03}=E_{0}
$$

such that for some integer $k$, each $E_{0(i+1)} / E_{0 i}$ splits as a direct sum of line bundles of total degree $k-i$.Then

(i) if $r k\left(E_{01}\right)>r k\left(E_{03} / E_{02}\right)$, the maximal subbundle $E_{b+}$ specializes to a direct summand of $E_{0}$ and of $E_{01}$ that is a direct sum of line bundles of total degree $k$;

(ii) if $r k\left(E_{01}\right)<r k\left(E_{0} / E_{02}\right)$, the minimal quotient $E_{b-}$ specializes to a direct summand of $E_{0} / E_{02}$ and of $E_{0}$ that is a quotient bundle that is a direct sum of line bundles of degree $k-2$;

(iii) if $r k\left(E_{01}\right)=r k\left(E_{0} / E_{02}\right)$, then $E_{b}$ is balanced.

Proof. Assertion (iii) is trivial and (i) and (ii) are mutually dual, so it will suffice to prove (i). To that end, note to begin with that by simple arithmetic, if we set

$$
r=\operatorname{rk}\left(E_{01}\right)-\operatorname{rk}\left(E_{03} / E_{02}\right)
$$

then the maximal subbundle

$$
E_{b+}=r \mathcal{O}(k)
$$


By unicity of the maximal subbundle $E_{b+}$, there exists a subsheaf of $E$ which restricts on the generic fibre $X_{b}$ to $E_{b+}$; let $E_{+}$be the saturation of such a subsheaf. Thus $E / E_{+}$is torsion-free, hence by elementary depth considerations, $E_{+}$is locally free, hence its restriction $E_{+0}$ on $X_{0}$ is a direct sum of $r$ line bundles, whose total degrees add up to $r k$.

Now let $S \subseteq E_{0+}$ be a line subbundle of maximal total degree. This degree is clearly at least $k$. Then $S^{*} \otimes E_{0}$ is a direct sum of line bundles of nonpositive total degree and admits a regular (locally nonzerodivisor) section. This clearly implies $S$ is a direct summand of $E_{0}$ (and of $E_{01}$ ) of degree exactly $k$. Therefore $E_{0+}$ is a direct sum of line bundles of total degree exactly $k$ and is a direct summand of $E_{0}$ and of $E_{01}$.

6. Normal bundles of generic rational curves and angles. A smooth rational curve or rational angle $C \subset \mathbb{P}^{n}$ is said to be almost balanced if its normal bundle is. The bidegree of a rational angle $C_{a} \cup C_{b} \subset \mathbb{P}^{n}$ is defined to be $(a, b)$. In this case, we recall that the notion of $C_{a}, C_{b}$ 'interfacing well' at the node $C_{a} \cap C_{b}$ was given in Definition 2.3 and some of its consequences given in Lemma 2.4. Our main purpose in this section is to prove the following result

THEOREM 6.1.

(i) (Sacchiero) A generic rational curve of degree $d \geq n$ in $\mathbb{P}^{n}$ is almost balanced.

(ii) Let

$$
C=C_{a} \cup C_{b} \subset \mathbb{P}^{n}
$$

be a generic rational angle of given bidegree $(a, b)$. Then $C_{a}, C_{b}$ interface well provided

$$
\max (a, b) \geq n
$$

As noted in the Introduction, part (i) is originally due to Sacchiero [S] by another, degeneration-free method. This method does not seem to yield part (ii), which we require. Before turning to the proof of Theorem 6.1 we note some explicit corollaries. First some notation. Fixing $n$, define integers $k(d), r(d)$ by

$$
(n+1) d-2=k(d)(n-1)+n-1-r(d), 0<r(d) \leq n-1 .
$$

Note that an almost balanced bundle of degree $-(n+1) d+2$ must have splitting type $\left((-k)^{r},(-k-1)^{n-1-r}\right)$, therefore

Corollary 6.2. (Sacchiero) A generic rational curve $C_{d}$ of degree $d \geq n$ in $\mathbb{P}^{n}$ has conormal bundle

$$
N^{*}=r(d) \mathcal{O}(-k(d)) \oplus(n-1-r(d)) \mathcal{O}(-k(d)-1) .
$$

We can similarly determine the splitting type of the normal bundle of generic rational angles.

Corollary 6.3. For a generic rational angle $C$ of bidegree $(a, b)$ in $\mathbb{P}^{n}$ with $a, b \geq n, C$ is almost balanced and we have:

Case 1: if $r(a)+r(b) \geq n+1$, then

$$
N_{C}^{*} \simeq(r(a)+r(b)-n-1) \mathcal{O}((-k(a)) \cup(-k(b))) \oplus
$$




$$
(n-r(b)) \mathcal{O}((-k(a)) \cup(-k(b)-1)) \oplus(n-r(a)) \mathcal{O}((-k(a)-1) \cup(-k(b))) ;
$$

Case 2: if $r(a)+r(b) \leq n$, then

$$
N_{C}^{*} \simeq(r(a)-1) \mathcal{O}((-k(a)) \cup(-k(b)-1)) \oplus(r(b)-1) \mathcal{O}((-k(a)-1) \cup(-k(b)))
$$

$$
\oplus(n+1-r(a)-r(b)) \mathcal{O}((-k(a)-1) \cup(-k(b)-1)) .
$$

Proof. From almost balancedness of $C_{a}, C_{b}$ it follows easily that, setting $N^{*}=$ $N_{C}^{*}$, we have

$$
\left.N^{*}\right|_{C_{a}} \simeq(r(a)-1) \mathcal{O}(-k(a)) \oplus(n-r(a)) \mathcal{O}(-k(a)-1)
$$

and likewise for $b$. Then almost balancedness of $C$ is equivalent to the positive subspaces of these bundles at the node $p$ being in general position. Since this holds by Thm 6.1, analyzing $N^{*}$ as in the proof of Proposition 5.1 and Example 5.5 yields the claimed splitting.

Corollary 6.4. For a generic rational angle $C$ of bidegree $(a, b)$ in $\mathbb{P}^{n}$ with $1 \leq a \leq n-1, n \leq b$, we have, setting $k=k(b)$ :

Case 1: if $r(b)>a$,

$$
\begin{gathered}
N_{C}^{*} \simeq(r(b)-a-1) \mathcal{O}((-a) \cup(-k)) \oplus \mathcal{O}((-a-1) \cup(-k)) \\
\oplus(n-r(b)) \mathcal{O}((-a) \cup(-k-1)) \oplus(a-1) \mathcal{O}((-a-2) \cup(-k)) ;
\end{gathered}
$$

Case 2: if $r(b) \leq a$

$$
\begin{aligned}
& N_{C}^{*} \simeq(n-a-1) \mathcal{O}((-a) \cup(-k-1) \oplus \mathcal{O}((-a-1) \cup(-k-1)) \\
& \oplus(r(b)-1) \mathcal{O}((-a-2) \cup(-k)) \oplus(a-r(b)) \mathcal{O}((-a-2) \cup(-k-1)) .
\end{aligned}
$$

In particular, if $a=n-1$ then $C$ is almost balanced. If $r(b)<a<n-1$ or $1<a<r(b)-1, C$ is not almost balanced.

Proof. Analogous to the preceding proof, again using the good interface of $C_{a}$ and $C_{b}$. Note that in the present case $C$ is not necessarily almost balanced unless $a=n-1$, so we cannot directly conclude from this that a general $C_{a+b}$ is almost balanced.

Note that putting together Theorem 6.1, Corollaries 6.2-6.4, Example 3.4 and Example 5.5 we now know the splitting type of the normal bundle of a generic rational curve or rational angle of every degree and bidegree.

The proof of Theorem 6.1 is somewhat long, so we break it into steps.

Step 1: Case $(\mathbf{n}, \mathbf{1})$. We begin by showing that $C$ is almost balanced if $a=n$, i.e. $C_{a}$ is a rational normal curve, and $b=1$, i.e. $C_{b}$ is a line $L$ meeting $C_{n}$. Applying a suitable projective transformation, we may assume that $C_{n}$ is the standard rational normal curve given parametrically in affine coordinates by

$$
x_{i}=t^{i}, i=1, \ldots, n,
$$

and that $L$ is a general line through the origin $p$. Note that if we project parallel to the coordinates $x_{4}, \ldots, x_{n}$ and prove almost balancedness for the projected curve, it 
will imply almost balancedness for the original; this is fairly clear a priori, and will become more clear with the computations that follow. Thus, it suffices to prove $C$ is almost balanced in case $n=3$. By semi-continuity, it would suffice to prove $C$ is almost balanced for one choice of $L$, and we pick the line with equation

$$
x_{1}-x_{3}=x_{2}=0 .
$$

Now recall the identification (see $\S 3$ )

$$
N_{C_{3}}^{*}=V_{1} \otimes \mathcal{O}(-5)
$$

As usual, the restriction $\left.N_{C}^{*}\right|_{C_{3}}$ is given by the elementary modification of $N_{C_{3}}^{*}$ corresponding to the Zariski conormal space to $C$ at $p$, and this conormal space is clearly generated by the class of $x_{2}$. Thus the positive subsheaf $H N_{1}\left(\left.N_{C}^{*}\right|_{C_{3}}\right)$ is the unique 'special' (i.e. in this case, degree- $(-5)$ ) subsheaf whose fibre at $p$ is generated by $x_{2}$ $\bmod \mathfrak{m}_{p, C_{3}}$, and clearly that subsheaf is generated locally by

$$
f=x_{2}-x_{1}^{2},
$$

which is none other than $\kappa_{\mathfrak{D}}$ where $\mathfrak{D}$ is the unique point at infinity on $C_{3}$, i.e. the point with homogeneous coordinates $[0,0,0,1]$. Now set

$$
g=x_{2}-x_{1} x_{2}
$$

and note that $f, g$ yield a local basis for $N_{C_{3}}^{*}$. Since $x_{1}$ is a local parameter on $C_{3}$, it also follows that a local basis for the elementary reduction $\left.N_{C}^{*}\right|_{C_{3}}$ is given by $f, x_{1} g$.

Now over on the $L$ side, it is easy to see that $\left.N_{C}^{*}\right|_{L}$ has local basis $x_{2}, x_{1}\left(x_{1}-x_{3}\right)$ with positive subsheaf $H N_{1}\left(\left.N_{C}^{*}\right|_{L}\right)$ generated by $x_{2}$. The claimed almost balancedness for $C$ means that the two positive subsheaves have different images in the fibre $N_{C}^{*} \otimes$ $k(p)$, which amounts to saying that we modify $f$ to another local section $f^{\prime}$ of $\left.N_{C}^{*}\right|_{C_{3}}$ that has the same fibre at $p$, and that lifts to a local function vanishing on $L$, viewing $f^{\prime}$ as a local section of $\left.N_{C}^{*}\right|_{L}$ and expressing it as a linear combination of $x_{2}, x_{1}\left(x_{1}-x_{3}\right)$, the coefficient of $x_{1}\left(x_{1}-x_{3}\right)$ is nonzero at $p$.

Indeed, set

$$
f^{\prime}=f+x_{3} g
$$

Since

$$
x_{3} \in \mathfrak{m}_{p, C_{3}}^{3},
$$

clearly $f$ and $f^{\prime}$ have the same image in $\left.N_{C}^{*}\right|_{C_{3}} \otimes k(p)$. On the other hand, we have

$$
f^{\prime}=\left(1-x_{1} x_{3}\right) x_{2}-\left(x_{1}+x_{3}\right)\left(x_{1}-x_{3}\right)
$$

so $f^{\prime}$ vanishes in $L$, and as section of $\left.N_{C}^{*}\right|_{L}$, we have

$$
f^{\prime}=\left(1-x_{1}^{2}\right) x_{2}-2 x_{1}\left(x_{1}-x_{3}\right),
$$

which proves our assertion.

Step 2: More on $(\mathbf{n}, \mathbf{1})$. Note that for $n=3$ what we have proven, in fact, is that

$$
N_{C}^{*} \simeq \mathcal{O}(-5 \cup-2) \oplus \mathcal{O}(-6 \cup-1)
$$


and in particular $N_{C}^{*}$ is balanced. For $n>3$, the positive subsheaf $P$ of $N_{C}^{*}$ has corank 2 (i.e. $\operatorname{rank}(n-3)$ ) and it is at this point easy- and worthwhile too- to identify explicitly its restriction on $C_{n}$. Now in terms of the identification (4.1), we have

$$
H N_{1}\left(\left.N_{C}^{*}\right|_{C_{n}}\right)=U \otimes \mathcal{O}(-n-2),
$$

for some codimension-1 subspace $U \subset V_{n-2}$. Clearly the intersection of $U$ with the open set of divisors $\mathfrak{D}$ not containing $p$ coincides with the set of such divisors such that

$$
L \subset \overline{\mathfrak{D}+2 p},
$$

because the quadric $K_{\mathfrak{D}}$ is nonsingular at $p$ with tangent hyperplane $\overline{\mathfrak{D}+2 p}$. Since both $U$ and the set of $\mathfrak{D}$ satisfying (6.6) are linear spaces, it follows that, in fact

$$
U=\{\mathfrak{D}: L \subset \overline{\mathfrak{D}+2 p}\} .
$$

Now clearly

$$
\left.P\right|_{C_{n}}=W \otimes \mathcal{O}(-n-2)
$$

where $W \subset U$ is a codimension- 1 subspace (codimension 2 in $V_{n-2}$ ). Checking again on divisors $\mathfrak{D}$ not containing $p$, note that if

$$
L \subset \overline{\mathfrak{D}+p}
$$

then the quadric $K_{\mathfrak{D}}$ is smooth at $p$ and contains $L$. Its equation at $p$, say $f$, on the one hand clearly yields a section of $H N_{1}\left(\left.N_{C}^{*}\right|_{C_{n}}\right)$; on the other hand, since the tangent hyperplane to $K_{\mathfrak{D}}$ at $p$ contains $L \cup T_{p} C_{n}$, it is also clear that $f$ yields a section of $H N_{1}\left(\left.N_{C}^{*}\right|_{L}\right)$. Thus $f$ in fact yields a section of $P$, so that

$$
\mathfrak{D} \in W .
$$

Since the set of $\mathfrak{D}$ with

$$
p \notin \mathfrak{D}, L \subset \overline{\mathfrak{D}+p}
$$

is itself an open set in a codimension-2 subspace of $V_{n-2}$ (or its projectivization) it follows that

$$
\{\mathfrak{D}: p \notin \mathfrak{D}, \mathfrak{D} \in W\}=\{\mathfrak{D}: p \notin \mathfrak{D}, L \subset \overline{\mathfrak{D}+p}\}
$$

hence, finally, that

$$
W=\{\mathfrak{D}: L \subset \overline{\mathfrak{D}+p}\} .
$$

The identity (6.8) has important consequences, including some general position or genericity properties. For convenience, let's temporarily set

$$
M=N_{C}^{*} \otimes k(p), S:=<T_{p} C_{n}, L>^{\perp}=T_{p} C^{\perp}
$$

where $T_{p}$ denotes the embedded Zariski tangent space, and note that $M$ contains 2 canonical hyperplanes, viz.

$$
U=H N_{1}\left(\left.N_{C}^{*}\right|_{C_{n}}, p\right)
$$

and its analogue from the $L$ side,

$$
U^{\prime}=H N_{1}\left(\left.N_{C}^{*}\right|_{L}, p\right),
$$


as well as the 1-dimensional 'singular' subspace $\sigma$ that is the kernel of either of the natural maps

$$
M \rightarrow N_{C_{n}}^{*} \otimes k(p), M \rightarrow N_{L}^{*} \otimes k(p) .
$$

We have shown that

$$
U \neq U^{\prime}
$$

and of course

$$
\sigma \notin U \cup U^{\prime} .
$$

Now note that $\left.N_{C}^{*}\right|_{L}$, being an elementary modification of $N_{L}^{*}$, and hence also $M$, depend only on the flag

$$
(p, L, S) .
$$

Consequently the group $G$ of projective motions preserving this flag acts on $M$, preserving $U^{\prime}, \sigma$. Moreover it is easy to see that $G$ acts transitively on the set of hyperplanes in $M$ different from $U^{\prime}$ and not containing $\sigma$. The upshot is that for given flag $(p, L, S), U$ may be assumed to be a generic hyperplane in $M$.

Another important general position property that follows from (6.8) is the following. Let $A \subset V_{n-2}$ be any irreducible subvariety. Then the locus

$$
\tilde{A}:=\left\{(\mathfrak{D}, p, L) \in A \times C_{n} \times \mathbb{G}(1, n): p \in L \subset \overline{D+p}\right\}
$$

maps to $A \times C_{n}$ so that every fibre is a $\mathbb{P}^{n-3}$. Therefore $\tilde{A}$ is irreducible and $(\operatorname{dim}(A)+$ $n-2)$-dimensional. Therefore the fibre of $\tilde{A}$ over a general pair $(p, L)$ with $p \in L$ is purely $(\operatorname{dim}(A)-2)-$ dimensional. When $A$ is a linear space, so is this fibre, and we conclude

Corollary 6.5. Given any linear subspace $A \subset V_{n-2}$, if $p \in C_{n}$ is sufficiently general and $L$ is a sufficiently general line through $p$, then $(p, L)$ impose independent conditions on $A$ in the sense that

$$
A(-(p, L)):=\{\mathfrak{D} \in A: L \subset \overline{\mathfrak{D}+p}\}
$$

is a codimension-2 subspace of $A$ if $\operatorname{dim}(A) \geq 2$ and zero if $\operatorname{dim}(A)=1$.

Translating this result into the language of normal bundles, we conclude the following.

Corollary 6.6. Assumptions as in Corollary 6.5, if $\operatorname{dim}(A) \geq 2$ (resp. $\operatorname{dim}(A)=$ 1) then, the intersection

$$
\left.A \otimes \mathcal{O}_{C_{n}}(-n-2) \cap N_{C_{n} \cup L}^{*}\right|_{C_{n}}
$$

performed inside $N_{C_{n}}^{*}$ and viewed as subsheaf of $\left.N_{C_{n} \cup L}^{*}\right|_{C_{n}}$, extends to a subbundle of $N_{C_{n} \cup L}^{*}$ of the form

$$
\begin{gathered}
A(-(p, L)) \otimes\left(\mathcal{O}_{C_{n}}(-n-2) \cup \mathcal{O}_{L}(-1)\right) \\
\oplus \mathcal{O}_{C_{n}}(-n-2) \cup \mathcal{O}_{L}(-2) \oplus \mathcal{O}_{C_{n}}(-n-3) \cup \mathcal{O}_{L}(-1)
\end{gathered}
$$

(resp.

$$
\left.\mathcal{O}_{C_{n}}(-n-3) \cup \mathcal{O}_{L}(-2)\right)
$$


Step 3: Case $(\mathbf{n}, \mathbf{1}, \mathbf{1})$. Now our strategy for the proof of the general case of Theorem 6.1 is essentially to degenerate (implicitly) a curve with 1 or 2 components in $\mathbb{P}^{n}$ to a chain consisting of lines, rational normal curves $C_{n}$ and some degenerate rational normal curves $C_{a}$, each lying in an $a$-plane in $\mathbb{P}^{n}$. To this end we consider next the case

$$
C=L_{1} \cup C_{n} \cup L_{2}
$$

where $L_{1}, L_{2}$ are generic lines among those incident to $C_{n}$ and meet it at generic points $p_{1}, p_{2}$, respectively. We will show that $N_{C}^{*}$ is decomposable and determine its decomposition as a direct sum of line bundles (no general assertion is made or needed about bundles on reducible curves with $>2$ components). We begin with the case $n=3$. In this case, note that

$$
\left.N_{C}^{*}\right|_{C_{3}} \simeq M \otimes \mathcal{O}(-6)
$$

where $M$ is a 2-dimensional vector space canonically isomorphic, up to scalars, to

$$
M_{i}=N_{C}^{*} \otimes k\left(p_{i}\right)=N_{C_{3} \cup L_{i}}^{*} \otimes k\left(p_{i}\right), i=1,2 .
$$

As above, $M_{i}$ contains a codimension- 1 subspace $U_{i}^{\prime}$ coming from the maximal subsheaf $\mathcal{O}(-1)$ on $L_{i}$ and, by the general position property discussed above, we may assume that under the identifications

$$
\mathbb{P}(M)=\mathbb{P}\left(M_{1}\right)=\mathbb{P}\left(M_{2}\right),
$$

we have

$$
U_{1}^{\prime} \neq U_{2}^{\prime}
$$

This clearly implies that

$$
N_{C}^{*} \simeq \mathcal{O}(-6 \cup-1 \cup-2) \oplus \mathcal{O}(-6 \cup-2 \cup-1) .
$$

Next consider the case $n=4$. Then with notations as above, we have hyperplanes

$$
U_{i}, U_{i}^{\prime} \subset M_{i}, i=1,2
$$

and a 1-dimensional subspace

$$
W_{i}=U_{i} \cap U_{i}^{\prime} .
$$

On the other hand we clearly have

$$
\left.N_{C}^{*}\right|_{C_{4}}=\mathcal{O}(-6,-7,-7)
$$

where the fibre $Z$ of the maximal subsheaf $\mathcal{O}(-6)$ coincides with $U_{1} \cap U_{2}$ under the natural embeddings

$$
U_{1}, U_{2} \subset V_{2} \text {. }
$$

Choosing things generally, we may clearly assume

$$
Z \not \subset U_{i}^{\prime}, i=1,2 .
$$

Therefore $N_{C}^{*}$ admits a direct summand of the form

$$
\mathcal{O}(-6 \cup-2 \cup-2) .
$$

Now let $a, b$ be a basis of $U_{1}^{\prime}$ and lift them to subsheaves

$$
A,\left.B \simeq \mathcal{O}(-7) \subset N_{C}^{*}\right|_{C_{4}},
$$


which is clearly possible. Modifying $A, B$ by the unique up to scalar map

$$
\left.\mathcal{O}(-7) \rightarrow \mathcal{O}(-6) \subset N_{C}^{*}\right|_{C_{4}}
$$

vanishing at $p_{1}$ we may assume

$$
A \otimes k\left(p_{2}\right), B \otimes k\left(p_{2}\right) \subset U_{2}^{\prime} .
$$

Then $A, B$ project $\bmod \mathcal{O}(-6)$ to generically, hence everywhere, linearly independent $\mathcal{O}(-7)$ subsheaves, and each of them glues to an $\mathcal{O}(-1)$ subsheaves on $L_{1}$ and $L_{2}$. Therefore finally

$$
N_{C}^{*} \simeq \mathcal{O}(-6 \cup-2 \cup-2) \oplus 2 \mathcal{O}(-7 \cup-1 \cup-1) .
$$

Finally consider the case $n \geq 5$. choosing $C_{n}, L_{1}, L_{2}$ generically, we get as above hyperplanes

$$
U_{i} \neq U_{i}^{\prime} \subset M_{i}, i=1,2
$$

where $U_{i}$ maps isomorphically to

$$
\left\{\mathfrak{D}: L_{i} \subset \overline{\mathfrak{D}+2 p_{i}}\right\} \subset V_{n-2},
$$

under the natural map

$$
\left.N_{C}^{*}\right|_{C_{n}} \rightarrow N_{C_{n}}^{*}
$$

and subspaces

$$
W_{i}=U_{i} \cap U_{i}^{\prime},
$$

which map isomorphically to

$$
\left\{\mathfrak{D}: L_{i} \subset \overline{\mathfrak{D}+p_{i}}\right\} \subset V_{n-2}, i=1,2 .
$$

as well as a codimension-2 subspace

$$
Z=U_{1} \cap U_{2} \subset V_{n-2}
$$

such that

$$
\left.N_{C}^{*}\right|_{C_{n}} \simeq Z \otimes \mathcal{O}(-n-2) \oplus 2 \mathcal{O}(-n-3) .
$$

Moreover

$$
Y=Z \cap W_{1} \cap W_{2}
$$

is of codimension 2 in $Z$ (hence vanishes if $n=5$ ). Analyzing as above, we conclude

$$
N_{C}^{*} \simeq Y \otimes \mathcal{O}(-n-2 \cup-1 \cup-1) \oplus
$$

$$
\mathcal{O}(-n-2 \cup-1 \cup-2) \oplus \mathcal{O}(-n-2 \cup-2 \cup-1) \oplus 2 \mathcal{O}(-n-3 \cup-1 \cup-1)
$$

Note that in (6.9) and (6.10) we have shown in particular that $N_{C}^{*}$ is decomposable (i.e. is a direct sum of line bundles) but have not used any assertion analogous to Proposition 5.1 for 3-component chains. 
Step 4: The critical range. Our next goal is to prove that $C_{d}$ is almost balanced in the critical range $n<d \leq 2 n$. This range is difficult because when $d$ is in it, a rational angle $C_{a, b}, a, b>1$ that is a limit of $C_{d}$ will usually not be almost balanced. Still, for $d=n+1, n+2$, note that almost balancedness of $C_{d}$ follows by specialization from almost balancedness of $C_{n} \cup L$ and $C_{n} \cup L_{1} \cup L_{2}$. In the general case we will work inductively. By specialization again, it will suffice to prove in the range $n+2 \leq d \leq 2 n-1$ that if $C_{d}$ is balanced, then so is a general connected union $C_{d} \cup L$. To this end, we will specialize $C_{d}$ to $C_{n} \cup C_{d-n}$ so that $L$ specialized to a unisecant of $C_{n}$. Set

$$
a=d-n \in[2, n-1], N^{*}=N_{C_{n} \cup C_{a}}^{*}
$$

and note that

$$
\begin{gathered}
\left.N^{*}\right|_{C_{n}} \simeq(n-2) \mathcal{O}(-n-2) \oplus \mathcal{O}(-n-3), \\
\left.N^{*}\right|_{C_{a}} \simeq(n-a-1) \mathcal{O}(-a) \oplus \mathcal{O}(-a-1) \oplus(a-1) \mathcal{O}(-a-2),
\end{gathered}
$$

where the positive subsheaf $(n-a-1) \mathcal{O}(-a) \subset N_{C_{a}}^{*}$ corresponds to the linear forms vanishing on $C_{a} \cup T_{p} C_{n}$. As we have seen, choosing the span of $C_{a}$ sufficiently generally, the fibre at $p$ of this positive subsheaf does not contain the fibre at $p$ of the positive subsheaf of $\left.N^{*}\right|_{C_{n}}$, and consequently we have, when $a \leq n-2$, a decomposition

$$
\begin{aligned}
& N^{*} \simeq(n-a-2) \mathcal{O}(-n-2 \cup-a) \oplus \mathcal{O}(-n-3 \cup-a) \\
& \oplus \mathcal{O}(-n-2 \cup-a-1) \oplus(a-1) \mathcal{O}(-n-2 \cup-a-2) .
\end{aligned}
$$

If $a=n-1$, we have a decomposition

$$
N^{*} \simeq \mathcal{O}(-n-3 \cup-n) \oplus(n-2) \mathcal{O}(-n-2 \cup-n-1)
$$

and in particular this bundle is (almost) balanced; this case is similar to but simpler than the case $a \leq n-2$, so assume the latter.

Now let $P$ be the positive subsheaf of $N_{C_{d}}^{*}$, which by almost balancedness is of the form $\rho \mathcal{O}(k)$, and let $P^{\prime}$ be the elementary modification of $P$ at a general point $q \in C_{d}$ corresponding to a general line $L$ through $q$, and $P_{+} \subset P^{\prime}$ its positive subsheaf, which is of the form $(\rho-1) \mathcal{O}(k)$ if $\rho>1$ and $(n-1) \mathcal{O}(k-1)$ if $\rho=1$. To prove that a $C_{d} \cup L$ is almost balanced, it would suffice to prove that if $\rho>1$ then the fibre at $q$ of $P_{+}$is not contained in the fibre at $q$ of the positive subsheaf of the elementary modification of $N_{L}^{*}$ corresponding to $T_{q} C_{d}$ (which corresponds to the set of hyperplanes containing $L \cup T_{q} C_{d}$, i.e. the Zariski conormal space to $C_{d} \cup L$ at $q$ ).

Now let $P_{0}$ be the limit of $P$ on $C_{n} \cup C_{a}$, as computed in Proposition 5.7. If $2 a<n-1$, then the restriction of $P_{0}$ on $C_{n}$ is of the form $\rho \mathcal{O}(-n-2)$ and our assertion follows by applying Corollary 6.6 to $C_{n}$ and $L$. If $2 a>n-1$, then $\left.P_{0}\right|_{C_{n}}$ contains a subbundle of the form $(2 a-1) \mathcal{O}(-n-2)$ and since we may assume $a>1$ our assertion again follows similarly from Corollary 6.6. If $2 a=n-1$ then $\left.P_{0}\right|_{C_{n}}$ contains $(n-2) \mathcal{O}(-n-2)$ so again we are done except if $n=3$, in which case $a=1$, against our assumption. (Alternatively, almost balancedness of $C_{n+a}$ and $C_{n+a} \cup L$ for $2 a<n-1$ could also be established by degenerating $C_{n+a}$ to $C_{n}$ plus $a$ many general unisecants, but we shall not need this.)

Let us say that a bundle $E$ on a curve $C$ is $b$-balanced, for an integer $b$, if it is a direct sum of line bundles $F_{i}$ whose (total) degrees on $C$ are contained in an interval of length $b$, i.e. $\max \operatorname{deg}\left(F_{i}\right)-\min \operatorname{deg}\left(F_{i}\right) \leq b$. Then in the above proof we have 
shown that $C_{n} \cup C_{a}$ is 2-balanced but not 1-balanced if $2 \leq a \leq n-3$, 1-balanced if $a=1$ or $n-1$ and 0 -balanced if $a=n-2$.

I claim next that

$$
C=C_{n-1} \cup L \subset \mathbb{P}^{n}
$$

is almost balanced. To see this, note that

$$
\begin{aligned}
\left.N_{C}^{*}\right|_{C_{n-1}} & \simeq \mathcal{O}(-n) \oplus(n-2) \mathcal{O}(-n-1), \\
\left.N_{C}^{*}\right|_{L} & \simeq(n-2) \mathcal{O}(-1) \oplus \mathcal{O}(-2)
\end{aligned}
$$

Where the positive subsheaf $\left.\mathcal{O}(-n) \subset N_{C}^{*}\right|_{C_{n-1}}$ is the chopped form of the subsheaf

$$
\mathcal{O}(-n+1) \subset N_{C_{n-1}}^{*},
$$

corresponding to the unique hyperplane containing $C_{n-1}$. Thus, the fibre at the node $p$ of this subsheaf is spanned by the singular element, and that fibre is not contained in the fibre at $p$ of the positive subsheaf of $\left.N_{C}^{*}\right|_{L}$. Therefore $C$ is almost balanced (even balanced) with normal bundle

$$
N_{C}^{*} \simeq \mathcal{O}(-n \cup-2) \oplus(n-2) \mathcal{O}(-n-1 \cup-1) .
$$

Step 5: A gluing lemma. Our proof of almost balancedness in higher degrees will be inductive, based largely on the following result

Lemma 6.7. Assume that

$$
C_{a} \cup L_{a}, C_{b} \cup L_{b} \subset \mathbb{P}^{n}
$$

are almost balanced, where $L_{a}, L_{b}$ denotes general unisecant lines to $C_{a}$ or $C_{b}$ respectively. Then

$$
C_{a} \cup L \cup C_{b} \subset \mathbb{P}^{n}
$$

is almost balanced, where $L$ denotes a general line meeting $C_{a}$ and $C_{b}$.

Proof. Set

$$
C=C_{a} \cup L \cup C_{b}, N^{*}=N_{C}^{*} .
$$

By almost balancedness of $C_{a} \cup L$, it is easy to see that $\left.N^{*}\right|_{C_{a} \cup L}$, which is a general elementary modification of $N_{C_{a} \cup L}^{*}$ at a general point of $L$, is of the form either (case $\mathrm{a} 0)$

$$
(\rho) \mathcal{O}(k \cup-1) \oplus 2 \mathcal{O}(k \cup-2) \oplus(n-3-\rho) \mathcal{O}(k-1 \cup-1), \rho \geq 0,
$$

or, if the positive subsheaf of $\left.N^{*}\right|_{C_{a}}$ is of rank 1 (case a1),

$$
\mathcal{O}(k \cup-2) \oplus(n-3) \mathcal{O}((k-1) \cup-1) \oplus \mathcal{O}((k-1) \cup-2)
$$

or, if $\left.N^{*}\right|_{C_{a}}$ coincides with its positive subsheaf, i.e. is balanced (case a2),

$$
(n-3) \mathcal{O}(k \cup-1) \oplus 2 \mathcal{O}(k \cup-2) .
$$

Likewise, we have analogous cases b0,b1,b2, where the integer analogous to $k$ will be denoted by $\ell$. Assume first that neither a1 nor b1 hold.

Note that the positive subsheaf of $\left.N^{*}\right|_{C_{a} \cup L}$, restricted on $L$, meets the positive subsheaf $(n-2) \mathcal{O}(-1)$ of $N^{*} \mid L$ in a subbundle of the form

$$
U_{a} \otimes \mathcal{O}(-1)=u_{a} \mathcal{O}(-1),
$$


likewise for $b$. Applying a suitable projective transformation to $C_{b}$, the subspaces

$$
U_{a}, U_{b} \subset H^{0}\left(I_{L}(1)\right)
$$

clearly may be assumed in general position. Then it is easy to see that if

$$
u_{a}+u_{b} \geq n-1
$$

then $N^{*}$ splits as

$$
\begin{gathered}
N^{*} \simeq\left(U_{a} \cap U_{b}\right) \otimes \mathcal{O}(k \cup-1 \cup \ell) \oplus \\
\nu_{a} \mathcal{O}(k \cup-1 \cup(l-1)) \oplus \nu_{b} \mathcal{O}((k-1) \cup-1 \cup \ell) \oplus 2 \mathcal{O}(k \cup-2 \cup \ell),
\end{gathered}
$$

where

$$
\nu_{a}=u_{a}-\operatorname{dim} U_{a} \cap U_{b}=n-1-u_{b},
$$

likewise for $\nu_{b}$. Note we do not need an analogue of Proposition 5.1 on $C$ for this. Otherwise, i.e. if

$$
u_{a}+u_{b}<n-1
$$

then $U_{a} \cap U_{b}=0$ and

$$
\begin{gathered}
N^{*} \simeq U_{a} \otimes \mathcal{O}(k \cup-1 \cup(\ell-1)) \oplus U_{b} \otimes \mathcal{O}((k-1) \cup-1 \cup \ell) \\
\oplus\left(n-3-u_{a}-u_{b}\right) \mathcal{O}((k-1 \cup-1 \cup(\ell-1)) \oplus \mathcal{O}(k \cup-2 \cup \ell) .
\end{gathered}
$$

If case a1 holds but b1 does not, we have a splitting

$$
\begin{gathered}
N^{*} \simeq U_{b} \otimes \mathcal{O}((k-1) \cup-1 \cup \ell) \\
\oplus\left(\left(n-3-u_{b}\right) \mathcal{O}((k-1) \cup-1 \cup(\ell-1)) \oplus \mathcal{O}(k \cup-2 \cup(\ell-1)) \oplus \mathcal{O}((k-1) \cup-2 \cup \ell) .\right.
\end{gathered}
$$

Likewise if b1 holds but a1 does not hold. Finally if a1 and b1 hold, then

$$
N^{*} \simeq(n-2) \mathcal{O}((k-1) \cup-1 \cup(\ell-1)) \oplus \mathcal{O}(k \cup-2 \cup(\ell-1)) \oplus \mathcal{O}((k-1) \cup-2 \cup \ell) .
$$

This completes the proof of Lemma 6.7. Again it is worth noting that although we have proven the decomposability of certain bundles (viz. $N_{C}^{*}$ ) on $C$, we did not require an analogue of Proposition 5.1, stating that all bundles on $C$ are decomposable.

Step 6: Conclusion. As one consequence of Lemma 6.7, we can now prove the almost balancedness of $C_{d}$ and $C_{d} \cup L$ in $\mathbb{P}^{n}$ for all $d \geq n$. The proof is by induction and the case $d \leq 2 n$ has been done previously. If $d>2 n$, specialize $C_{d}$ to $C_{n} \cup M \cup C_{d-n-1}$ and $L$ to a general unisecant of $C_{n}$. By Lemma 6.7 and induction, $C_{n} \cup M \cup C_{d-n-1}$ is almost balanced. Moreover considering the known results about $C_{n} \cup M \cup L$, it is easy to see that $L \cup C_{n} \cup M \cup C_{d-n}$ is almost balanced as well. Therefore $C_{d}$ and $C_{d} \cup L$ are almost balanced, completing the induction step.

Next we show that

$$
C_{a} \cup C_{b} \subset \mathbb{P}^{n}
$$

is almost balanced whenever

$$
n-1 \leq a, n \leq b .
$$

Indeed, it suffices to degenerate $C_{a} \cup C_{b}$ to $C_{a} \cup L \cup C_{b-1}$ and use the fact that $C_{a} \cup L$ and $L \cup C_{b-1}$ are almost balanced, together with Lemma 6.7. 
To complete the proof of Theorem 6.1 it now suffices to show that $C_{a}, C_{b}$ interface well when

$$
C=C_{a} \cup C_{b}, \quad 2 \leq a \leq n-2, b \geq n
$$

Then we have

$$
\begin{gathered}
\left.N^{*}\right|_{C_{a}} \simeq(n-1-a) \mathcal{O}(-a) \oplus \mathcal{O}(-a-1) \oplus(a-1) \mathcal{O}(-a-2), \\
\left.N^{*}\right|_{C_{b}} \simeq(r(b)-1) \mathcal{O}(-k) \oplus(n-r(b)) \mathcal{O}(-k-1), k=k(b) .
\end{gathered}
$$

If $r(b)=1$, good interface is automatic so assume $r(b)>1$. As usual, set $M=$ $N^{*} \otimes k(p)$ and also let $L=T_{p} C_{a}$ which, vis-a-vis $C_{b}$, is a general unisecant at $p$. Then $M$ splits naturally as

$$
M=<\sigma>\oplus S
$$

where $S$ may be canonically identified with the set of linear froms vanishing on $L \cup$ $T_{p} C_{b}$. Now

$$
S_{1}:=H N_{1}\left(\left.N^{*}\right|_{C_{a}}, p\right)
$$

may be identified with the set of linear form vanishing on $C_{a} \cup T_{p} C_{b}$, or equivalently on $\mathbb{P}^{a} \cup T_{p} C_{b}$ where $\mathbb{P}^{a}$ is the linear span of $C_{a}$, hence for fixed $S$ and $C_{b}, S_{1}$ may be considered a generic $(n-1-a)$-dimensional subspace of $S$. On the other hand, in proving $C_{b} \cup L$ is almost balanced for a generic line $L$ through $p$ we showed that

$$
H N_{1}\left(\left.N^{*}\right|_{C_{b}}, p\right) \not \subset S \text {. }
$$

Therefore

$$
S_{2}:=S \cap H N_{1}\left(\left.N^{*}\right|_{C_{b}}, p\right)
$$

is $(r(b)-2)$-dimensional and meets $S_{1}$ transversely within $S$, and therefore $S_{1}$ and $H N_{1}\left(\left.N^{*}\right|_{C_{b}}, p\right)$ meet transversely within $M$.

Next, $H N_{2}\left(\left.N^{*}\right|_{C_{a}}, p\right)$ is spanned by $S_{1}$ and $\sigma$ and since $S$ maps isomorphically to $M / \sigma$ and $S_{1}$ is generic in $S$, it follows the the image of $H N_{2}\left(\left.N^{*}\right|_{C_{a}}\right) \otimes k(p)$ in $M / \sigma$ is a generic $(n-d-1)$-dimensional subspace, hence meets the image of $H N_{1}\left(\left.N^{*}\right|_{C_{b}}\right) \otimes k(p)$ transversely. Thus $C_{a}$ and $C_{b}$ interface well at $p$.

7. Remarks on degenerating linear systems. The purpose of this section is to work out a convenient local model for a specialization of a linear system as $\mathbb{P}^{1}$ specializes to a rational angle. Consider, in $\mathbb{P}^{1} \times \mathbb{P}^{1} \times \mathbb{A}^{1}$ with coordinates $([U],.[V], s$.$) ,$ the divisor $X$ (of type $(1,1))$ with equation

$$
U_{1} V_{1}=s U_{0} V_{0} .
$$

It is immediate that $X$ is a smooth surface and the projection

$$
\pi: X \rightarrow B:=\mathbb{A}^{1}
$$

is flat with fibres $\pi^{-1}(s)=\mathbb{P}^{1}, s \neq 0$ and

$$
X_{0}=\pi^{-1}(0)=X_{1} \cup X_{2}=\left(V_{1}=0\right) \cup\left(U_{1}=0\right)
$$

a rational angle. Moreover either one of the projections $X \rightarrow \mathbb{P}^{1} \times \mathbb{A}^{1}$ is a blowing up in one point. Set

$$
L=p_{\mathbb{P}^{1} \times \mathbb{P}^{1}}^{*}(\mathcal{O}(a, b)) .
$$


Then $L$ is a line bundle with degree $d=a+b$ on a fibre of $\pi$ and bidegree $(a, b)$ on $X_{0}$. Moreover it is immediate from the defining equation (7.1) that $\pi_{*}(L)$ is a trivial bundle of rank $d+1$ with basis

$$
U_{0}^{a} V_{0}^{b}, U_{0}^{a_{0}} U_{1}^{a-a_{0}} V_{0}^{b}, U_{0}^{a} V_{0}^{b_{0}} V_{1}^{b-b_{0}}, a_{0}=0, \ldots, a-1, b_{0}=0, \ldots b-1,
$$

or in affine coordinates $u=U_{1} / U_{0}, v=V_{1} / V_{0}$,

$$
1, u, \ldots, u^{a}, v, \ldots, v^{b} .
$$

It will be important for our purposes to determine the 'dualizing principal parts' sheaf $P_{X / B}^{+}(L)$ (cf. (2.4)), which coincides with the locally free double dual of $P_{X / B}^{1}(L)$ :

LEMma 7.1. We have a linear isomorphism

$$
\nabla^{\ell}=\left(\nabla_{1}, \nabla_{2}\right): P_{X / B}^{+}(L) \stackrel{\sim}{\rightarrow} \mathcal{O}_{X}(a-1, b) \oplus \mathcal{O}_{X}(a, b-1)
$$

which, via the inclusion $P_{X / B}^{1}(L) \subset P_{X / B}^{+}(L)$, corresponds to the differential operator $\nabla$ on $L$ which on relative global sections is given by

$$
\begin{aligned}
\nabla^{h}: & F \mapsto\left(\partial F / \partial U_{0}+s V_{0} / U_{1} \partial F / \partial V_{1}, \partial F / \partial V_{0}+s U_{0} / V_{1} \partial F / \partial U_{1}\right) \\
& =\left(\partial F / \partial U_{0}+V_{1} / U_{0} \partial F / \partial V_{1}, \partial F / \partial V_{0}+U_{1} / V_{0} \partial F / \partial U_{1}\right)
\end{aligned}
$$

and which in affine coordinates $(u, v)$ at $p$ is given by

$$
\nabla^{a}: f \mapsto(a f-u \partial f / \partial u+v \partial f / \partial v, b f+u \partial f / \partial u-v \partial f / \partial v)
$$

Proof. To begin with, it is easy to check that (7.3) and (7.4) are in fact equal, dehomogenize to (7.5) and that they vanish on $F_{0}=U_{1} V_{1}-s U_{0} V_{0}$, hence send a multiple of $F_{0}$ to another multiple of $F_{0}$. Therefore they define an $\mathcal{O}_{B}$-linear, $\mathcal{O}_{X}(a-$ $1, b) \oplus \mathcal{O}_{X}(a, b-1)$-valued differential operator $\nabla$ on $L$ over $X$, a priori a rational one but from the equality of (7.3) and (7.4) clearly $\nabla$ is regular. Hence it defines a linear map $\nabla^{\ell}$ on $P_{X / B}^{1}(L)$. This map takes the value $(a, b)$ on $F=U_{0}^{a} V_{0}^{b}$, i.e. $f=1$. Now this section $f=1$ gives rise to a trivialization of $L$ and hence to a splitting, locally near the node $p$,

$$
P_{X / B}^{1}(L) \simeq \mathcal{O}_{X} \oplus \Omega_{X / B}, P_{X / B}^{+}(L) \simeq \mathcal{O}_{X} \oplus \omega_{X / B}
$$

By (7.5), we have

$$
\nabla^{\ell}(d u)=(-u, u), \nabla^{\ell}(d v)=(v,-v) .
$$

Now the dualizing sheaf $\omega_{X / B}$ is generated locally at $p$ by a form $\eta$ equal to $d u / u$ on $X_{1}$ and to $-d v / v$ on $X_{2}$, and clearly

$$
\nabla^{\ell}(\eta)=(-1,1)
$$

Since $(a, b)=\nabla^{\ell}(1)$ and $(-1,1)$ form a local basis for the target of $\nabla^{\ell}$, it follows that $\nabla^{\ell}$ extends to $P_{X / B}^{+}(L)$ to yield an isomorphism as stated in the Lemma, locally near $p$. It is easy to check that $\nabla^{\ell}$ is an isomorphism locally at any non-critical point, therefore it is an isomorphism. 
8. Smoothing the normal bundle of a rational angle. In $\S 6$ we determined the normal bundle to a generic rational angle

$$
C_{a, b} \subset \mathbb{P}^{n}
$$

and saw, in particular, that it is often not almost balanced when

$$
\min (a, b)<n .
$$

As a result, the locus of these rational angles will appear as an improper part of the locus of curves of degree $d=a+b$ with unbalanced normal bundle. For our enumerative purposes, this locus must therefore be subtracted off, with the correct multiplicity, to get the correct count of smooth curves with unbalanced normal bundle. Our next goal, then, is the determination of these multiplicities. This will require a study of the deformation of the normal bundle in a smoothing of $C_{a, b}$, which we will do using the methods of the previous section.

Now fix natural numbers $n, a, b$ with $b \leq a, b<n$, set $d=a+b$ and consider a general local linear system of rank $(n+1)$ and bidegree $(a, b)$, which we may consider extended to a subbundle $W$ of $\pi_{*}(L)$ defined near 0 . As a relative linear system on $X / B, W$ is clearly very ample on $X_{0}$, with image a general rational angle $C_{a, b} \subset \mathbb{P}^{n}$. Therefore $W$ is relatively very ample in a neighborhood of $X_{0}$ and embeds a general fibre $X_{s}$ as a smooth rational curve of degree $d$ in $\mathbb{P}^{n}$, thus providing an explicit smoothing of $C_{a, b}$. We denote by

$$
f=f_{W}: X \rightarrow \mathbb{P}^{n}
$$

the associated mapping, defined near $X_{0}$. Working in an affine neighborhood of the node $p=([1,0],[1,0])$ of $X_{0}$, we use coordinates $u=U_{1} / U_{0}, v=V_{1} / V_{0}$.

Now let $N$ denote the relative lci normal bundle for $f$ relative to $\pi$ and $\mathbb{N}=$ $N \otimes L^{-1}$ as usual. Fix a line bundle $\tau$ of bidegree $(r+1,1)$ on $X_{0}$ (note that $\tau$ is in fact unique), and set

$$
\begin{gathered}
r=\left\lceil\frac{2 d-2}{n-1}\right\rceil-1, \\
N_{0}=\mathbb{N}^{-1}(-\tau) .
\end{gathered}
$$

We say that the pair $(d, n)$ is perfect if $(n-1) \mid 2(d-1)$. The enumerative results of $\S 9$ will apply only to perfect pairs. The main result of this section is

Proposition 8.1. (Cohomological quasitransversality) Notations as above, if $(d, n)$ is perfect, then for the generic smoothing $W, R^{1} \pi_{*}\left(N_{0}\right)_{0}$ is killed by $\mathfrak{m}_{0, B}$ and is a $\underline{k}(0)$ - vector space of dimension $b-1$.

Remark. The proof will show more generally, in the not-necessarily-perfect case, that $R^{1} \pi_{*}\left(N_{0}\right)_{0}$ is a direct sum of $(b-1) \underline{k}(0)$ and a free part of rank $(n-1)(r+1)-$ $(2 d-2)$.

Proof. To begin with, let us dispense with the easy case where $N_{0}$ is almost balanced. In that case, $N_{0}$ itself is a direct sum of line bundles $K$, and the only ones which contribute to $R^{1}$ are $\mathcal{O}(-2,0)$ and $\mathcal{O}(-1,-1)$, whose $R^{1}$ is easily seen to be free; moreover these summands cannot occur at all in the perfect case. 
Turning now to the proof proper, it will be based on the exact sequence (2.5) which in our case takes the form

$$
0 \rightarrow N^{*}(L) \rightarrow W \otimes \mathcal{O}_{X} \rightarrow \mathcal{O}(a-1, b) \oplus \mathcal{O}(a, b-1) \rightarrow q \rightarrow 0
$$

with $q$ a (generic) length-1 skyscraper sheaf at $p$, and which dualizes to

$$
0 \rightarrow \mathcal{O}(-a+1,-b) \oplus \mathcal{O}(-a,-b+1) \rightarrow W^{*} \otimes \mathcal{O}_{X} \rightarrow \mathbb{N} \rightarrow q \rightarrow 0 .
$$

Twisting (8.2) by $\mathcal{O}(-r-1,-1)$ yields

$0 \rightarrow \mathcal{O}(-a-r,-b-1) \oplus \mathcal{O}(-a-r-1,-b) \stackrel{\phi}{\rightarrow} W^{*} \otimes \mathcal{O}_{X}(-r-1,-1) \rightarrow N_{0} \rightarrow q \rightarrow 0$.

Now by Theorem 6.1 we have for the generic smoothing that

$$
\pi_{*}\left(N_{0}\right)=0,
$$

hence $R^{1} \pi_{*}\left(N_{0}\right)$ consists of a locally free part of rank $(n-1)(r+1)-(2 d-2)$ plus some torsion at 0 . Moreover, it is easy to check from the results of $\S 6$ that

$$
h^{1}\left(\left.N_{0}\right|_{X_{0}}\right)=((n-1)(r+1)-(2 d-2))+(b-1)
$$

or equivalently,

$$
h^{0}\left(\left.N_{0}\right|_{X_{0}}\right)=b-1
$$

(note that $h^{0}\left(\left.N_{0}\right|_{X_{1}}\right)=0$ ). Therefore the value given by Proposition 8.1 is, in an obvious sense, the smallest possible value for $R^{1} \pi_{*}\left(N_{0}\right)$ - which explains the term 'cohomological quasitransversality', and consequently by semi-continuity it will suffice to exhibit one smoothing $W$ for which the assertion of Proposition 8.1 holds.

The following Commutative Algebra assertion will allow us to reduce to the case $r \leq 5$ :

Lemma 8.2. Let $A$ be a regular local ring and $s, t$ part of of a regular system of parameters on $A$. Let $M$ be an $A$ - module of finite type such that for some natural number $n$,

(i) $M / t M \simeq n A /(t)$;

(ii) $M / s M \simeq n A /(s, t)$.

Then $t M=0$ or equivalently, $M \simeq n A /(t)$.

Proof of Lemma. Use induction on $n$, which coincides with the number of minimal generators of $M$. Suppose first that $n=1$, so $M=A / I$. By (i), we have $I \subseteq(t)$, so write $I=t J$. Then by (ii), we have $(t J, s)=(t, s)$ so clearly $J=(1)$ as desired.

In the general case pick a primary cyclic submodule

$$
N=A u \simeq A / Q \subseteq M,
$$

where $u \in M$ is a suitable minimal generator. Since $u$ maps to minimal generators of $M / t M, M / s M$, clearly hypotheses (i), (ii) are inherited by the quotient $M / N$, so by induction we have

$$
M / N \simeq(n-1) A /(t) .
$$

Now if $Q \not \subset(t)$, then $t$ is regular (i.e. multiplication by it is injective) on $N=A / Q$ and consequently the kernel of multiplication by $t$ on $M$ itself maps isomorphically to 
$M / N=(n-1) A /(t)$. Therefore we get a splitting

$$
M \simeq(n-1) A /(t) \oplus N .
$$

So $N$, as quotient of $M$, inherits properties (i), (ii) and hence by the case $n=1$ already considered, we have $N \simeq A /(t)$, contradiction.

Therefore we may assume $Q \subset(t)$ hence, as $Q$ is primary, $Q=\left(t^{m}\right)$ for some $m \geq 1$. Since $s$ is regular on $M / N$, we get an injection

$$
N / s N \simeq A /\left(t^{m}, s\right) \hookrightarrow M / s M \simeq n A /(s, t)
$$

which clearly forces $m=1$. Now since $t$ kills $N$ and $M / N$ and $N$ is saturated, being generated by a minimal generator $u$ of $M$, it follows that $t$ kills $M$ so by (i), $M \simeq$ $n A /(t)$. The Lemma is proved.

Corollary 8.3. If Proposition 8.1 holds for all $a \leq 2 n-2$ then it holds for all $a$.

Proof. By induction, suppose that $a>2 n-2$ and that the Proposition holds for all $a^{\prime}<a$. Consider a general curve of the form

$$
C=C_{n-1} \cup_{q} C_{a-n+1} \cup_{p} C_{b} .
$$

Consider a 2-parameter smoothing of this curve parametrized by $s, t$ where $s=0$ (resp. $t=0$ ) is the locus where $q$ (resp. $p$ ) remains singular. Consider a general smoothing of the appropriate linear system, and let $\mathbb{N}$ be the relative normalized normal bundle and set

$$
N_{0}=\mathbb{N} \otimes L^{-1} \otimes \mathcal{O}((-2) \cup(-r+1) \cup(-1))
$$

(i.e. $\mathbb{N}$ twisted by a line bundle with the appropriate degrees on the respective components of $C$ ). Now the results of $\S 6$ show that as $C_{a}$ specializes to $C_{a-n+1} \cup C_{n-1}$, the cohomology of $\mathbb{N}$ remains constant: in fact $N_{C_{a-n+1} \cup C_{n-1}}$ splits as a direct sum of line bundles in such a way that the splitting deforms, summand by summand, to a splitting of $N_{C_{a}}$ (cf. Remark 5.4.1). This implies that the cohomology of $N_{0}$ is constant along the locus $t=0$ where $p$ remains a node (in this locus the general curve is of the form $C_{a} \cup C_{b}$ and the special one is $C_{n-1} \cup C_{a-n+1} \cup C_{b}$ ). By induction, Proposition 8.1 holds for $C_{a-n+1} \cup C_{b}$, and then it follows easily that the restriction of the cohomology of $N_{0}$ (i.e. $R^{1} \pi_{*}\left(N_{0}\right)$, whose formation clearly commutes with basechange, being an $R^{\mathrm{top}} \pi_{*}$ ) on the locus $s=0$ where $q$ remains a node is annihilated by $t$, since the corresponding assertion holds for the smoothing of $C_{a-n+1} \cup C_{b}$. Applying the Lemma and restricting to a slice $s=\epsilon$ gives a smoothing as desired for Proposition 8.1. This proves the Corollary.

We are therefore reduced to considering the case $a \leq 2 n-2$ which implies $d \leq$ $3 n-3$ and therefore $r \leq 5$.

Chopping (8.3) into short exacts, we see that Proposition 8.1 will hold provided the cokernel of $R^{1}\left(\pi_{*} \phi\right)$ is of the form

$$
\operatorname{coker}\left(R^{1}(\phi) \simeq b k(0) \oplus((n-1)(r+1)-(2 d-2)) \mathcal{O}_{B, 0}\right.
$$

(note that if (8.4) holds then $\pi_{*}\left(N_{0}\right)=0$ for the given smoothing $W$ ). Now $R^{1}\left(\pi_{*} \phi\right)$ is a map of free $\mathcal{O}_{B}$-modules, and it is easy to see that (8.4) holds iff the dual $\mu=$ 
$R^{1} \pi_{*}(\phi)^{*}$ satisfies

$$
\operatorname{coker}(\mu) \simeq b k(0) .
$$

Now set

$$
V_{c, d}=\pi_{*}(\mathcal{O}(c, d)) .
$$

Then written out explicitly, using standard duality identifications and Lemma 7.1, $\mu$ comes out as the map

$$
\begin{aligned}
W \otimes V_{r, 0} & \rightarrow V_{a+r-1, b} \oplus V_{a+r, b-1}, \\
\chi \otimes g & \mapsto\left(g \nabla_{1}(\chi), g \nabla_{2}(\chi)\right)
\end{aligned}
$$

with $\left(\nabla_{1}, \nabla_{2}\right)$ as in Lemma 7.1. We must show a choice of $W$ for which the image of $\mu$ is of the form $s z_{1}, \ldots, s z_{b}, z_{b+1}, \ldots, z_{2(d+r)}$ where $(z$.) is a basis for the target of $\mu$ and $s$ is the parameter on $B$.

Now let us introduce some convenient notation. Set

$$
e=(a, b), e[m]=(a-m, b+m) .
$$

Then it is easy to see that

$$
\begin{aligned}
& \nabla\left(u^{i}\right)=\left(\nabla_{1}\left(u^{i}\right), \nabla_{2}\left(u^{i}\right)\right)=e[i] u^{i}, \\
& \nabla\left(v^{i}\right)=e[-i] v^{i} .
\end{aligned}
$$

Now we claim that the desired property (8.5) will hold for $W$ with basis $\chi_{0}, \ldots, \chi_{n}$ as follows: $\chi_{0}=1, \chi_{1}=u$ and for each $2 \leq i \leq n$, either

$$
\chi_{i}=u^{\nu_{i}}
$$

(which we call a $u$-move), or

$$
\chi_{i}=u^{\nu_{i}}+v^{\rho_{i}}
$$

(which we call a $v$-move), where the choice of the exponents $\nu_{i}, \rho_{i}$ is to be specified. Note that a basis for the source of $\mu$ is given by

$$
\chi_{i} \otimes u^{j}, i=0, \ldots, n, j=0, \ldots, r,
$$

and we view the $\mu\left(\chi_{i} \otimes u^{j}\right)$ as arranged naturally in a $(r+1) \times(n+1)$ matrix $\Theta$ and as a totally ordered set, column by column. We denote by $\Theta_{i}$ the submatrix of $\Theta$ containing the first $i+1$ columns. One choice of basis for the target of $\mu$ is given by

$$
\begin{gathered}
e[i(j)] u^{j}, e\left[i^{\prime}(j)\right] u^{j}, j=0, \ldots, a+r-1, e[a] u^{a+r}, \\
e[k(j)] v^{j}, e\left[k^{\prime}(j)\right] v^{j}, j=1, \ldots, b-1, e[-b] v^{b},
\end{gathered}
$$

as long as $i(j) \neq i^{\prime}(j), k(j) \neq k^{\prime}(j), \forall j$ (which implies that $e[i(j)], e\left[i^{\prime}(j)\right]$ (resp. $\left.\left.e[k(j)], e\left[k^{\prime}(j)\right]\right)\right)$ are linearly independent. Our aim is to devise a 'winning strategy' for the choice of $\nu_{i}$ and $\rho_{i}$ 's, which by definition means that we have

$$
\begin{gathered}
\operatorname{im}(\mu)=\operatorname{span}\left(e, k^{2} u, \ldots, k^{2} u^{a+r-1}, e[a] u^{a+r}, e[-1] v, \ldots, e[-b] v^{b},\right. \\
\left.s e[-1], s e[-2] v, \ldots, s e[-b] v^{b-1}\right)=: \Xi
\end{gathered}
$$

(span meaning over $\mathcal{O}_{B}$ ), which would clearly imply (8.5). That $\operatorname{im}(\mu) \subseteq \Xi$ is trivial and will emerge in the ensuing discussion. 
To this end, set

$$
U=V_{r, 0}, W_{i}=\operatorname{span}\left(\chi_{0}, \ldots, \chi_{i}\right) .
$$

Note that $\mu\left(W_{1} \otimes U\right)$ contains

$$
\operatorname{span}(e[i], e[i-1]) u^{i}=\operatorname{span}\left(k^{2} u^{i}\right), i=1, \ldots, r
$$

but only a 1-dimensional subspace of $k^{2} u^{0}, k^{2} u^{r+1}$. Accordingly, we set

$$
x_{1}=r, z_{1}=1
$$

Generally, we will say that an entry $\theta_{i j}=u^{k}+* v^{*}$ of $\Theta$ or a power $u^{k}$ is doubled if

$$
\mu\left(W_{i} \otimes U\right) \supset k^{2} u^{k}
$$

to show (8.7) holds it is of course sufficient- and in practice necessary- to show that $e[j] u^{k}, e\left[j^{\prime}\right] u^{k}$ occur in $\Theta_{i}, j \neq j^{\prime}$. Now suppose that

$$
\nu_{0}=0, \nu_{1}=2, \ldots, \nu_{i-1}
$$

have been defined, where $i \leq n$, in such a way that for each $c<i$, the set of $j$ such that $\mu\left(W_{c} \otimes U\right)$ contains (resp. meets nontrivially) $k^{2} u^{j}$ forms an integer interval $\left[1, x_{c}\right]$ (resp. $\left.\left[0, y_{c}\right]\right)$; indeed $y_{c}=\max \left(\nu_{d}: d \leq c\right)+r$. We will specify a uniquely determined way of choosing $\nu_{i+1}$, in case of a $u$-move, or $\left(\nu_{i+1}, \rho_{i+1}\right)$, in case of a $v$-move. This will reduce our problem of proving (8.6) to that of devising a suitable strategy of choosing at each stage a $u$ or $v$ move, where the total number of $u$ (resp. $v$ ) moves is $n-1-b$ (resp. $b$ ). We call such a sequence of moves allowable.

For a $u$-move, define

$$
\begin{gathered}
\nu_{i}^{\prime}=x_{i-1}+1, \\
\nu_{i}=\min \left(\nu_{i}^{\prime}, a\right), \\
x_{i}=\min \left(y_{i-1}, \nu_{i}+r\right), y_{i}:=\max \left(\nu_{d}: d \leq i\right)+r .
\end{gathered}
$$

We call the case $\nu_{i}^{\prime}>a$ an overboard case. It is then clear that $\mu\left(W_{i} \otimes U\right)$ contains (resp. meets nontrivially) $k^{2} u^{j}$ iff $j \in\left[1, x_{i}\right]$ (resp. $j \in\left[0, y_{i}\right]$ ), and that a $u$-move essentially always enlarges the rank of $\mu(W \otimes U)$ by $r+1$ : more precisely

$$
\operatorname{rk}\left(\mu\left(W_{i} \otimes U\right)\right)=\min \left(\operatorname{rk}\left(\mu\left(W_{i-1} \otimes U\right)+r+1,2(d+r)\right) .\right.
$$

In the case of a $v$-move, define

$$
\begin{gathered}
\nu_{i}^{\prime}=x_{i-1}-1, \\
\nu_{i}=\min \left(\nu_{i}^{\prime}, a\right),
\end{gathered}
$$

and $x_{i}, y_{i}$ as in the $u$ case. Here again an overboard case is where $\nu_{i}^{\prime}>a$. As for $\rho_{i}$, we simply define

$$
\rho_{i}=\max \left(\rho_{c}: c<i\right)+1
$$

where the max is over those $c$ so that $\rho_{c}$ is defined (i.e. so that the $c$ th move is a $v$-move), or 0 if no such $c$ exist. Thus the $\rho$ 's which are defined simply take the values $1, \ldots, b$. Note that for a $v$-move, the $i$-th column of $\Theta$ takes the form (transposed)

$$
\left(e_{0} u^{x_{i-1}-1}+e_{1} v^{\rho_{i}}, e_{0} u^{x_{i-1}}+s e_{1} v^{\rho_{i}-1}, \ldots\right)
$$


where $e_{0}=e\left[x_{i-1}-1\right], e_{1}=e\left[-\rho_{i}\right]$. For the first two entries in the vector, the $u$ term in in $\mu\left(W_{i-1} \otimes U\right)$ by definition, and consequently

$$
e_{1} v^{\rho_{i}}, s e_{1} v^{\rho_{i}-1} \in \mu\left(W_{i} \otimes U\right) .
$$

From the third entry on, the $v$ parts, which take the form

$$
s^{2} e_{1} v^{\rho_{i}-2}, \ldots, s^{\rho_{i}} e_{1}, \ldots, s^{\rho_{i}} e_{1} u^{r-\rho_{i}}
$$

in case $\rho_{i} \leq r$, or

$$
s^{2} e_{1} v^{\rho_{i}-2}, \ldots, s^{r} e_{1} v^{\rho_{i}-r}
$$

in case $\rho_{i} \geq r$, are clearly in $\mu\left(W_{i-1} \otimes U\right)$, so we conclude that

$$
e_{0} u^{x_{i-1}+1}, \ldots, e_{0} u^{x_{i-1}+r-1} \in \mu\left(W_{i} \otimes U\right) .
$$

It is clear in any case that as long as $x_{i-1}+1 \leq a$ (u move) or $x_{i-1}-1 \leq a$ ( $v$ move), the entries of $\Theta_{i}$ form part of a basis of $\Xi$.

Now it is easy to see from the definition that we always have

$$
\nu_{i} \leq x_{i} \leq \nu_{i}+r .
$$

Consequently,

$$
\nu_{i} \geq \nu_{i-1}
$$

except in the one case where the $i$ th column is a $v$-move and $x_{i-1}=\nu_{i-1}$ (we call this an exceptional $v$-move), in which we have

$$
\nu_{i}=\nu_{i-1}-1, x_{i}=\nu_{i}+r .
$$

Accordingly, the top $u$ power occurring in $\Theta$ through the $i$ th column always occurs in the $i$ th column itself, except if the $i$ th column is an exceptional $v$-move, in which the top $u$ power occurs in the $(i-1)$ st column.

It will be useful to introduce the notion of $i$ th level $z_{i}$, defined as follows: if $\nu_{i} \geq$ $\nu_{i-1}, z_{i}$ is the number of non-doubled elements in the $i$ th column, i.e. $z_{i}=\nu_{i}+r-x_{i}$; if $\nu_{i}=\nu_{i-1}-1$, set $z_{i}=-1$. Levels transform nicely under $u$ and $v$ moves: define

$$
\phi(x)=r+1-|x|, \psi(x)=r-1-|x| .
$$

Then after a $u$ move in the $i$ th column, $z_{i}=\phi\left(z_{i-1}\right)$, while after a $v$ move in the $i$ th column, $z_{i}=\psi\left(z_{i-1}\right)$. Note that $\phi, \psi$ are essentially reflections, i.e.

$$
\phi^{2}(x)=\psi^{2}(x)=|x| .
$$

Now a key observation is the following:

Lemma 8.4. Suppose $\Theta$ is constructed by an allowable sequence of moves and $z_{n}=1$. Then (8.6) holds, i.e. we have a winning strategy.

To see this, suppose first there are no overboards. Then the fact that $z_{n}=1$ implies that the powers $u, \ldots, u^{\nu_{n}+r-1}$ are all doubled and having $b$ many $v$-moves ensures that

$$
e[-i] v^{i}, s e[-i-1] v^{i} \in \operatorname{im}(\mu), i=0, \ldots, b-1 .
$$

Also $e\left[\nu_{n}\right] u^{\nu_{n}+r}, e[-b] v^{b} \in \operatorname{im}(\mu)$, obviously. Since

$$
(r+1)(n+1) \geq 2(d+r)=2 a+2 b+2 r,
$$


this is only possible if $\nu_{n}=a$, so (8.6) holds. Now if an overboard occurs at step $i$, it is easy to check that $\nu_{j} \geq a \forall j \geq i$ and using $\nu_{n}=a$ we can conclude as above.

Recalling that $z_{1}=1$ automatically, we are thus reduced to finding an allowable word $w$ in $\phi, \psi$, i.e. one containing $b$ many $\psi$ 's and $n-1-b$ many $\phi$ 's, so that $w(1)=1$. This is easiest if $n$ is odd: ideed in this case it suffices to set

$$
\begin{gathered}
w=\psi^{b} \phi^{n-1-b}, b \text { even, } \\
w=\phi^{n-2-b} \psi^{b} \phi=\phi^{n-2-b} \psi^{b-1}(\psi \phi), b \text { odd }
\end{gathered}
$$

(using $\psi \phi(1)=-1$ ).

Henceforth we assume that $n$ is even. Recall from Corollary 8.3 that we may assume $2 \leq r \leq 5$. Since we are assuming $(d, n)$ is perfect, we have

$$
(r+1)(n-1)=2 d-2,
$$

therefore $r$ must be odd, i.e. $r=3$ or 5 . Suppose first that

$$
r=3 .
$$

This implies

$$
\psi( \pm 1)=1
$$

So it suffices to take

$$
\begin{gathered}
w=\phi^{n-1-b} \psi^{b}, b \text { odd } \\
w=\phi^{n-2-b} \psi^{b} \phi=\phi^{n-2-b} \psi^{b-1}(\psi \phi), b \text { even. }
\end{gathered}
$$

Next, suppose

$$
r=5 .
$$

Note that if $b=n-1$ then $a \geq n-1$; if strict inequality holds, then $C_{a, b}$ is almost balanced by the results of $\S 6$, while if equality holds then $r=4$ (and $(d, n)$ cannot be perfect). Therefore we may assume

$$
2 \leq b \leq n-2 .
$$

Then note

$$
\psi \phi \psi(1)=1
$$

so we can take

$$
\begin{gathered}
w=\phi^{n-2-b} \psi^{b-1} \phi \psi, b \text { even, } \\
w=\phi^{n-b-3} \psi^{b-2}(\phi \psi)^{2}, b \text { odd }
\end{gathered}
$$

(note that $b \leq n-2, b$ odd, $n$ even forces $n-b \geq 3$ ).

Remark 8.4. In the foregoing argument, our only use of the assumption that $(d, n)$ is perfect was to avoid the cases $r=2,4$. However, with a fairly short additional argument that we now sketch, the proof can be extended to cover those cases as well. This involves taking advantage of the 'slack' due to the fact that $(r+1)(n+1)>$ $2(d+r)$, i.e. the source of $\mu$ has larger rank than its asserted image. Indeed, define a map $\mu^{\prime}$ analogous to $\mu$ but with the exponents $\nu_{i}^{\prime}$ in place of $\nu_{i}$. Then, it is easy to check that we have a winning strategy (for $\mu$ ) if we can arrange for $\mu^{\prime}$ to have its $n$th level $z_{n}^{\prime}$ be either 0,1 or 2 . The latter can be achieved with the following choices. 
For $r=2($ so $\phi(1)=2), \psi(1)=0)$ :

$$
\begin{aligned}
& b \text { even : } w=\phi^{n-1-b} \psi^{b} \Rightarrow z_{n}^{\prime}=2 \\
& b \text { odd : } w=\psi^{b} \phi^{n-1-b} \Rightarrow z_{n}^{\prime}=0 .
\end{aligned}
$$

For $r=4($ so $\phi(2)=3, \psi(1)=2)$ :

$$
\begin{gathered}
b \text { odd : } w=\phi^{n-1-b} \psi^{b} \Rightarrow z_{n}^{\prime}=2, \\
b \text { even : } w=\psi \phi^{n-1-b} \psi^{b-1} \Rightarrow z_{n}^{\prime}=1 .
\end{gathered}
$$

Remark 8.5. Clearly condition (8.6), which is sufficient for the conclusion of Proposition 8.1 to hold, depends on $W$ only modulo $s^{2}$, i.e. depends only on the angle $C_{a, b}$ and its first-order deformation corresponding to $W \bmod s^{2}$.

Remark 8.6. Although the proof of Proposition 8.1 as written uses Theorem 6.1, it would be relatively straightforward to rewrite the proof to make it independent of the latter, thus providing another, albeit more algebraic and less geometric proof of Theorem 6.1.

9. Enumerative results. We are now able to state and prove our main enumerative results on rational curves whose normal bundles are not almost balanced. This will be done mainly by combining the cohomological computations of $\S 8$ with the (Grothendieck) Riemann-Roch formula, using as well some known qualitative and enumerative results concerning a generic 'incidence pencil' of rational curves, i.e. a 1parameter family defined by incidence to a generic collection of rational curves, which are summarized in the Appendix, whose notation and results we shall be using freely.

Thus let

$$
\pi: X \rightarrow B
$$

be a generic incidence pencil as in the Appendix, i.e. a smooth model of a generic 1-parameter family of rational curves of degree $d$ in $\mathbb{P}^{n}$ incident to a generic collection (A.) of linear spaces. Let

$$
f: X \rightarrow \mathbb{P}^{n}
$$

be the natural map, and set $L=f^{*} \mathcal{O}(1)$. We choose an ordering on the set of components $X_{1}, X_{2}$ of each reducible fibre of $\pi$, so that

$$
a:=L \cdot X_{1} \geq b:=L \cdot X_{2},
$$

and we call such a fibre of bidegree $(a, b)$. We assume henceforth that $(d, n)$ is a perfect pair and set, as in $\S 8$

$$
r=\frac{2 d-2}{n-1}-1 .
$$

A twisting divisor $D$ on $X$ is by definition an integral divisor on $X$ such that

(i) for a fibre $F$ of $\pi, D . F=r+2$;

(ii) for a reducible fibre $X_{1} \cup X_{2}$ of bidegree $(a, b)$,

$$
\text { D. } X_{1}=r+1, D \cdot X_{2}=1 .
$$


It is clear that twisting divisors exist; a specific choice is given by

$$
D=(r+1) s_{1}-\sum_{X_{2} \in \mathcal{F}_{1}} X_{2}-r \sum_{X_{1} \in \mathcal{F}_{1}} X_{1},
$$

where $\mathcal{F}_{1}$ is the set of fibre components not meeting $s_{1}$.

We fix a twisting divisor $D$ and let $\mathbb{N}$ be the relative normalized normal bundle of $X / B$, and set

$$
G=\mathbb{N}(-D) .
$$

Then the restriction of $G$ on a smooth fibre $X_{b}$ of $\pi$ has degree $-(n-1)$. In fact for the generic $b, f\left(X_{b}\right)$ is a generic rational curve of degree $d$ in $\mathbb{P}^{n}$ and hence by Theorem 6.1 , we have

$$
\left.G\right|_{X_{b}} \simeq(n-1) \mathcal{O}(-1) .
$$

The smooth fibres $X_{b}$ for which (9.2) does not hold are precisely those for which

$$
h^{1}\left(\left.G\right|_{X_{b}}\right)>0 .
$$

Accordingly, we call the corresponding curve $C=f\left(X_{b}\right)$ a normally jumping rational curve of multiplicity $h=h^{1}\left(\left.G\right|_{X_{b}}\right)$. For the generic pencil, the multiplicity $h$ depends only on $C$ itself.

THEOREM 9.1. With the above notations, the number of normally jumping curves in the pencil $B(a$.$) , weighted according to multiplicity, is given by$

$$
\begin{gathered}
J_{n, d}^{\perp}(a .)= \\
L\left(L+K_{X / B}\right)-D\left(\frac{n-3}{2} K_{X / B}+\frac{n-1}{2} L\right)-2 N_{d}^{\mathrm{red}}(a .)-\sum(b-1) N_{a, b}(a .),
\end{gathered}
$$

the summation being over all $a+b=d, b \leq a, 1<b<n$.

Proof. Applying (-1) times Grothendieck-Riemann-Roch ([F], Thm. 15.2; see [R5], Thm. 3.1 for a similar computation) to the sheaf $G$ and the mapping $\pi$, we get an equation, one side of which is the total length of $R^{1} \pi_{*}(G)$, since by the above discussion $\pi_{*}(G)=0$ and $R^{1} \pi_{*}(G)$ is of finite length. By Proposition 8.1, the length in question is the sum of the sought-for weighted number of normally jumping curves and the $\sum(b-1) N_{a, b}(a$. $)$ term from (9.3). To complete the proof it suffices to evaluate the other side of -GRR, which is routine. Briefly, the general formula of [F] yields

$$
-\int_{X}\left(\left((n-1) 1_{X}+c_{1}(G)+\frac{1}{2}\left(c_{1}^{2}-2 c_{2}\right)(G)\right)\left(1_{X}-\frac{1}{2} K_{X}+\chi\left(\mathcal{O}_{X}\right)[\mathrm{pt}]\right)\right)_{2}
$$

where $[\mathrm{pt}]$ is a point and $(\cdot)_{2}$ denotes the part in degree 2 . Note that $K_{X}=\omega+(2 g-2) F$ where $\omega=K_{X / B}, F$ is a fibre of $\pi$ and $g$ is the genus of $B$. Now the Chern classes of $\mathbb{N}$ can be computed from the exact sequence (8.1), yielding

$$
\begin{gathered}
c_{1}(\mathbb{N})=2 L+\omega, \\
c_{2}(\mathbb{N})=3 L(L+\omega)+\omega^{2}-\sigma
\end{gathered}
$$


where $\sigma=N_{d}^{\text {red }}(a$.) is the number of singular points of (fibres of) $\pi$, which by (A15) equals $-\omega^{2}$. Therefore

$$
\left(c_{1}^{2}-2 c_{2}\right)(\mathbb{N})=-L^{2}-2 L \omega-3 \sigma .
$$

Then standard Chern calculus yields

$$
\left.\frac{-1}{2}\left(c_{1}^{2}-2 c_{2}\right)(G)=L^{2}+L \omega-\frac{3}{2} \sigma+(2 L+\omega) D\right)-\frac{n-1}{2} D^{2} .
$$

For the other two product terms of (9.4), note that because $X$ is a blown-up $\mathbb{P}^{1}$-bundle, we have $\chi\left(\mathcal{O}_{X}\right)=\chi\left(\mathcal{O}_{B}\right)=1-g$ and by $(9.2), c_{1}(G) . F=-(n-1)$, hence

$$
-\chi\left(\mathcal{O}_{X}\right)+\frac{1}{2} K_{X} c_{1}(G)=\frac{1}{2} \omega(2 L+\omega-(n-1) D) .
$$

Then summing (9.5) and (9.6) and subtracting $\sum(b-1) N_{a, b}(a$.) yields (9.3).

Appendix: rational curves in $\mathbb{P}^{n 1}$. The purpose of this appendix is to review some notations and results, both qualitative and enumerative, about rational curves in $\mathbb{P}^{n}$ that are used in the statement and proof of the main result. Proofs and further details may be found in $[\mathrm{R} 2, \mathrm{R} 3, \mathrm{R} 4]$ and references therein.

We begin by reviewing some qualitative results about families of rational curves in $\mathbb{P}^{n}$, especially for $n \geq 3$. See [R2][R3] [R4] and references therein for details and proofs. In what follows we denote by $\bar{V}_{d}$ the closure in the Chow variety of the locus of irreducible nonsingular rational curves of degree $d$ in $\mathbb{P}^{n}, n \geq 3$, with the scheme structure as closure, i.e. the reduced structure (recall that the Chow form of a reduced 1-cycle $Z$ is just the hypersurface in $G\left(1, \mathbb{P}^{3}\right)$ consisting of all linear spaces meeting $Z)$. Thus $\bar{V}_{d}$ is irreducible reduced of dimension

$$
\operatorname{dim}\left(\bar{V}_{d}\right)=(n+1) d+n-3 .
$$

Let

$$
A_{1}, \ldots, A_{k} \subset \mathbb{P}^{n}
$$

be a generic collection of linear subspaces of respective codimensions $a_{1}, \ldots, a_{k}, 1 \leq$ $a_{i} \leq n$. We denote by

$$
B=B_{d}=B_{d}(a .)=B_{d}(A .)
$$

the normalization of the locus (with reduced structure)

$$
\left\{\left(C, P_{1}, \ldots, P_{k}\right): C \in \bar{V}_{d}, P_{i} \in C \cap A_{i}, i=1, \ldots, k\right\},
$$

and refer to it as a (generic) incidence family or incidence subvariety of $\bar{V}_{d}$. If all $a_{i}>1$ then this locus is also the normalization of its projection to $\bar{V}_{d}$, i.e. the locus of degree- $d$ rational curves (and their specializations) meeting $A_{1}, \ldots, A_{k}$. We have

$$
\operatorname{dim} B=(n+1) d+n-3-\sum\left(a_{i}-1\right) .
$$

When $\operatorname{dim} B=0$ we set

$$
N_{d}(a .)=\operatorname{deg}(B)
$$

\footnotetext{
${ }^{1}$ This Appendix is reproduced with a few modifications from [R1] for the reader's convenience
} 
Note that $N_{d}(1, a)=.d N_{d}(a$. $)$, which allows us to reduce the computation of the general $N_{d}\left(a\right.$.) to the case where all $a_{i}>1$, in which case we will say the conditionvector $\left(a\right.$.) is proper. The number $k$ of $a_{i}$ such that $a_{i}>1$ is called the length of the condition-vector $(a$.). Whenever $b=\operatorname{dim}(B) \geq 0$, it is convenient to set

$$
N_{d}^{\prime}(a .)=N_{d}(a ., b+1)
$$

and note that this is the degree in $\mathbb{P}^{n}$ of the locus swept out by the curves in $B(a$.).

The numbers $N_{d}$ and $N_{d}(a$.), first computed in general by Kontsevich and Manin (see for instance $[\mathrm{FP}]$ and references therein), are computed in [R2],[R3] by an elementary method, reviewed below, based on recursion on $d$ and $k$.

Now suppose $B=B(a$.) is such that $\operatorname{dim} B=1$ and $(a$.) is proper and let

$$
\pi: X \rightarrow B
$$

be the normalization of the tautological family of rational curves, and

$$
f: X \rightarrow \mathbb{P}^{n}
$$

the natural map. We call $B$ or $X / B$ a (generic) incidence percil. The following summarizes results from $[\mathrm{R} 2][\mathrm{R} 3][\mathrm{R} 4]$ :

Theorem A0. (i) $X$ is smooth .

(ii) Each fibre $C$ of $\pi$ is either

(a) a $\mathbb{P}^{1}$ on which $f$ is either an immersion with at most one exception which maps to a cusp $(n=2)$ or an embedding $(n>2)$; or

(b) a pair of $\mathbb{P}^{1}$ 's meeting transversely once, on which $f$ is an immersion with nodal image $(n=2)$ or an embedding $(n>2)$; or

(c) if $n=3$, a $\mathbb{P}^{1}$ on which $f$ is a degree-1 immersion such that $f\left(\mathbb{P}^{1}\right)$ has a unique singular point which is an ordinary node.

(iii) If $n>2$ then $\bar{V}_{d, n}$ is smooth along the image $\bar{B}$ of $B$, and $\bar{B}$ is smooth except, in case some $a_{i}=2$, for ordinary nodes corresponding to curves meeting some $A_{i}$ of codimension 2 twice. If $n=2$ then $\bar{V}_{d, n}$ is smooth in codimension 1 except for a cusp along the cuspidal locus and normal crossings along the reducible locus, and $\bar{B}$ has the singularities induced from $\bar{V}_{d, n}$ plus ordinary nodes corresponding to curves with a node at some $A_{i}$, and no other singularities.

Next, we review some of the enumerative apparatus introduced in [R3][R4] to study $X / B$. Set

$$
m_{i}=m_{i}(a .)=-s_{i}^{2}, i=1, \ldots, k .
$$

Note that if $a_{i}=a_{j}$ then $m_{i}=m_{j}$. It is shown in [R2] [R3][R4] that these numbers can all be computed recursively in terms of data of lower degree $d$ and lower length $k$. For $n \geq 2$, note that

$$
s_{i} . s_{j}=N_{d}\left(\ldots, a_{i}+a_{j}, \ldots, \hat{a}_{j}, \ldots\right), i \neq j .
$$

Also, letting $R_{\ell}$ denote the sum of all fibre components not meeting $s_{\ell}$, we have

$$
s_{j} \cdot R_{\ell}=\sum N_{d_{1}}^{\prime}\left(a_{i}: i \in I\right) N_{d_{2}}^{\prime}\left(a_{i}: i \notin I\right) .
$$


the summations being over all $d_{1}+d_{2}=d$ and all index-sets $I$ with $j \in I, \ell \notin I$, so all these numbers may be considered known. Then we have

$$
m_{i}=\frac{1}{2}\left(s_{i} \cdot R_{j}+s_{i} \cdot R_{p}-s_{j} \cdot R_{p}\right)-s_{i} \cdot s_{j}-s_{i} \cdot s_{p}+s_{j} \cdot s_{p}
$$

for any distinct $i, j, p$, and the RHS here is an expression of lower degree and/or length, hence may be considered known.

Next, set

$$
L=f^{*}(\mathcal{O}(1))
$$

and note that

$$
L^{2}=N_{d}(2, a .), L . s_{i}=N_{d}\left(a_{1}, \ldots, a_{i}+1, \ldots\right), i=1, \ldots, k
$$

(in particular, $L . s_{i}=0$ if $a_{i}=n$.) We computed in [R3] that, for any $i$,

$$
L \sim d s_{i}-\sum_{F \in \mathcal{F}_{i}} \operatorname{deg}(F) F+\left(N_{d}\left(a_{1}, \ldots, a_{i}+1, \ldots\right)+d m_{i}(a .)\right) F_{0}
$$

where $F_{0}$ is the class of a complete fibre and $\mathcal{F}_{i}$ is the set of fibre components not meeting $s_{i}$. Consequently we have

$$
N_{d}\left(2, a_{1}, \ldots\right)=2 d N_{d}\left(a_{1}+1, a_{2}, \ldots\right)+d^{2} m_{1}(a .)-\sum_{F \in \mathcal{F}_{1}(a .)}(\operatorname{deg} F)^{2}
$$

and clearly the RHS is a lower degree/length expression, so all the $N_{d}(2, \ldots)$ are known. We also have for $n>2$ that

$$
\begin{gathered}
N_{d}\left(a_{1}, a_{2}+1, \ldots\right)-N_{d}\left(a_{1}+1, a_{2}, \ldots\right)= \\
d N_{d}\left(a_{1}+a_{2}, \ldots\right)-\sum_{F \in\left(\mathcal{F}_{1}-\mathcal{F}_{2}\right)(a .)}(\operatorname{deg} F)+N_{d}\left(a_{1}+1, a_{2}, \ldots\right)+d m_{1}(a .)
\end{gathered}
$$

and again the RHS here is 'known', hence so is the LHS, which allows us to 'shift weight' between the $a_{i}$ 's till one of them becomes equal to 2 , so we may apply (A7), and thus compute all of the $N_{d}(a$.)'s.

Next, it is easy to see as in [R3] that

$$
L . R_{j}=\sum d_{2} N_{d_{1}}^{\prime}\left(a_{i}: i \in I\right) N_{d_{2}}\left(a_{i}: i \notin I\right), n \geq 2
$$

the summation for $n>2$ being over all $d_{1}+d_{2}=d$, and all index-sets $I$ such that $j \in I$.

Finally, the relative canonical class $K_{X / B}=K_{X}-\pi^{*}\left(K_{B}\right)$ was computed in [R3] as

$$
K_{X / B}=-2 s_{i}-m_{i} F+R_{i}
$$

for any $i$. Note that $-R_{i}^{2}$ equals the number $\sigma$ of reducible (equivalently, singular) fibres in the family $X / B$, a number we denote by $N_{d}^{\text {red }}(a$.), and which is easily computable by recursion, namely let

$$
N_{d_{1}, d_{2}}=\left(\begin{array}{c}
3 d-2 \\
3 d_{1}-1
\end{array}\right) d_{1} d_{2} N_{d_{1}} N_{d_{2}}, n=2
$$




$$
N_{d_{1}, d_{2}}\left(a_{.}\right)=\sum_{I} N_{d_{1}}^{\prime}\left(a_{i}: i \in I\right) N_{d_{2}}^{\prime}\left(a_{i}: i \notin I\right), n \geq 2,
$$

where the latter sum extends over all index-sets $I$. Then

$$
N_{d}^{\mathrm{red}}(a .)=\sum_{d_{1}+d_{2}=d} N_{d_{1}, d_{2}}\left(a_{\text {. }}\right) .
$$

From this we compute easily that

$$
\begin{gathered}
L \cdot K_{X / B}=-2 N_{d}\left(\ldots a_{i}+1 \ldots\right)-d m_{i}+L \cdot R_{i}, \\
K_{X / B}^{2}=-N_{d}^{\mathrm{red}}(a .) .
\end{gathered}
$$

\section{REFERENCES}

[EV1] D. Eisenbud, A. VAn De Ven, On the normal bundle of smooth space curves, Math. Ann., 256 (1981), pp. 453-463.

[EV2] - On the variety of smooth rational space curves with given degree and normal bundle, Invent. math., 67 (1982), pp. 89-100.

[F] W. Fulton, Intersection theory, Springer, 1984.

[FP] W. Fulton and R. Pandharipande, Notes on stable curves and quantum cohomology, AMS, Proceedings, Santa Cruz 1995.

[GS] F. Ghione, G. Sacchiero, Normal bundles of rational curves in $\mathbb{P}^{3}$., Manuscr. Math., 33 (1980), pp. 111-128.

[H] R. Hartshorne, Algebraic Geometry, Springer, 1977.

[OSS] Ch. Okonek, M. Schneider, H. Spindler, Vector bundles on projective spaces, Birkhäuser, 1980.

[Ram] L. Ramella, La stratification du schéma de Hilbert des courbes rationelles de $\mathbb{P}^{n}$ par le fibré tangent restreint, Comptes Rendus Acad. Sci. Paris Sér. I, 311 (1990), pp. 181-184.

[R1] Z. RAN, Geometry on nodal curves (to appear in Compositio Math. 2005).

[R2] —, Bend, break and count, Isr. J. Math, 111 (1999), pp. 109-124.

[R3] — Bend, break and count II, Math. Proc. Camb. Phil . Soc., 127 (1999), pp. 7-12.

[R4] - On the variety of rational space curve, Isr. J. Math, 122 (2001), pp. 359-370.

[R5] — The degree of the divisor of jumping rational curves, Quart. J. Math. (2001), pp. 118.

[R6] - Enumerative geometry of divisorial families of rational curves, Ann. Sc. Norm. Sup. Pisa Cl. Sci ser. 5, 3 (2004), pp. 67-85.

[S] G. Sacchiero, Normal bundles of rational curves in projective space, Ann. Univ. Ferrara Sez. VII, 26 (1981), pp. 33-40. 